\title{
Decision Procedures for Elementary \\ Sublanguages of Set Theory. \\ XI. Multilevel Syllogistic Extended by Some Elementary Map Constructs
}

D Cantone
J. Schwartz

Technical Report 374

May 1988

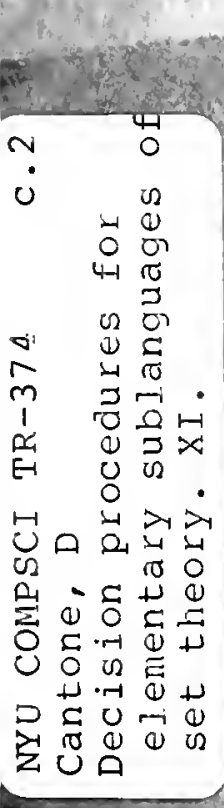




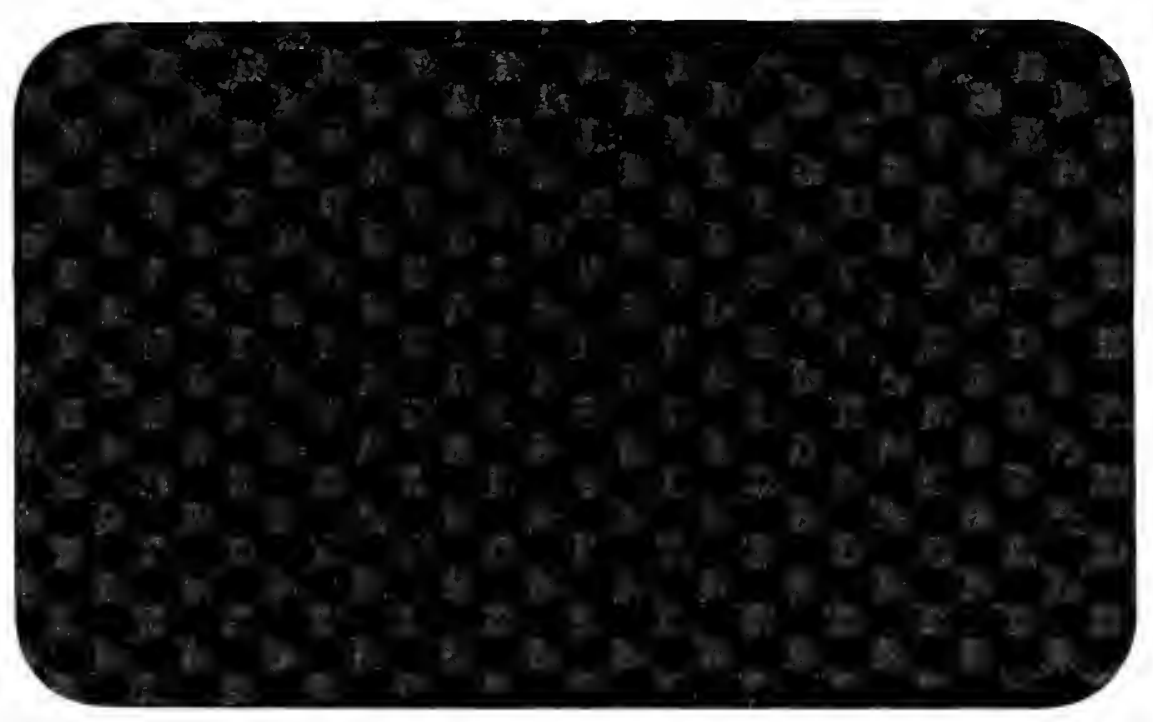


Decision Procedures for Elementary

Sublanguages of Set Theory.

$X I$. Multilevel Syllogistic Extended by

Some Elementary Map Constructs

D. Cantone

J. Schwartz

Technical Report 374

May 1988 



\section{DECISION PROCEDURES FOR ELEMENTARY SUBLANGUAGES OF SET THEORY. XI. MULTILEVEL SYLLOGISTIC EXTENDED BY SOME ELEMENTARY MAP CONSTRUCTS \\ D. CANTONE \\ Computer Science Department, Courant Institute of Mathematical Sciences, New York, New York 10012; and \\ Mathematics Department, University of Catania, Catania, Italy and \\ J.T. Schwartz \\ Computer Science Department, Courant Institute of Mathematical Sciences, New York, New York 10012}

\section{INTRODUCTION AND PRELIMINARIES}

This paper extends earlier work (with the same general title) on decision procedures for various quantified and unquantified languages of set theory (see [Can] for an extensive bibliography on this subject). We consider the language $\mathcal{L}$ built using the elementary Boolean connectives (conjunction, disjunction, implication, negation) from set-theoretic clauses of the forms

$$
\begin{aligned}
& x=y \cup z, x=y \backslash z, x \in y, d=D f \\
& \operatorname{PAIR} \operatorname{IN}(x, y, f), \operatorname{INV}(f, g), \operatorname{SINGLEVALUED}(f)
\end{aligned}
$$

The intended meaning of the operator $D$ and predicates PAIR_IN, INV, SINGLEVALUED is the following: write the subset of $f$ consisting of all ordered 
pairs in $f$ as pairs $(f)$, so that each $p \in \operatorname{pairs}(f)$ has the form $p=[x, y]$ (below we will be more specific on the set representation of $[x, y]$ ). The term $D f$, designates the domain of $f$, i.e., the set $\{x:[x, y] \in$ pairs $(f)$, for some $y\}$. The predicate PAIR_IN $(x, y, f)$ is true if and only if $[x, y] \in \operatorname{pairs}(f)$. Given sets $f$ and $g$, we assume that $\operatorname{INV}(f, g)$ holds if and only if pairs $(f)=\left((\operatorname{pairs}(g))^{-1}\right.$, i.e. for each pair $[p, q],[p, q]$ belongs to $f$ if and only if the inverse pair $[q, p]$ belongs to $g$. Notice that according to our definition, each $f$ admits an entire class of inverses, e.g. ( $\operatorname{pairs}(f))^{-1} \cup S$, as $S$ ranges over the sets which contain no pairs. Concerning the predicate SINGLEVALUED $(f)$, we assume that it holds whenever the relation pair $(f)$ is singlevalued, i.e. $f$ contains no two distinct pairs of the form $[s, t]$ and $\left[s, t^{\prime}\right]$. Finally, the intended meaning of the standard operators and predicates appearing in (1) is the usual one. Hence a model $M$ of a set $P$ of sentences of $\mathcal{L}$ is a function which maps every variable $x$ appearing in $P$ into an "ordinary" set $M x$ (of the standard universe of naive set theory). (See [CFS] for a somewhat more extended discussion of this point.) The ordered pair notion can of course be represented by any one of several more primitive set-theoretic constructions. To complete all details of the proof which is to follow, we need to choose one such, so for specificity we will define the ordered pair notion by

$$
[x, y]=\{\{0,\{x\}\},\{2,\{y\}\}\}
$$

where the integers 0 and 2 have definitions given by von Neumann, namely $0=\emptyset, 2=\{\emptyset,\{\emptyset\}\}$. Hence a set is an ordered pair if it has exactly two elements $e_{0}, e_{2}$, each of which is a pair. Exactly one of these, namely $e_{0}$ (resp. $e_{2}$ ) must have 0 (resp. 2) as an element; and the other element of each must be a singleton. It is then plain that the first component $x$ and the second component $y$ of $[x, y]$ can be recovered as the elements of these uniquely characterized singletons.

[FOS] solves the satisfiability problem for the three-sorted language which consists of set operators $U, \backslash$, set predicates $=, \epsilon$, cardinality operators \#, + , cardinality predicates $=,<, \leq$, function operators $D$ (domain), $R$ (range), $f[t]$ (where $f$ is a function variable and $t$ is a set term), and function predicates singlevalued $(f)$, one-one $(f)$. Notice that in the above language constructs like $f_{1}=f_{2} \cup f_{3}, f_{1}=f_{2} \backslash f_{3}, x \in f_{1}, f_{1} \in f_{2}$, PAIR_IN $(x, y, f)$, etc., where $f_{1}, f_{2}, f_{3}$ are function variables and $x, y$ are set variables, are forbidden.

This paper tackles the satisfiability problem for the one-sorted language $\mathcal{L}$, which properly extends the purely set-theoretical part (i.e. with no cardinality constructs) of the theory considered in [FOS]. In particular we will exhibit a finite and uniform procedure which decides for any given formula $P$ of $\mathcal{L}$ whether $P$ has a model or not. 
By way of disjunctive normal form, it is immediate to see that the satisfiability problem for $\mathcal{L}$ reduces to the satisfiability problem for the subfamily of conjunctions of atoms or negation of atoms of type (1). In fact we can limit ourselves without loss of generality to considering simple conjunctions of positive clauses of the form (1). To this purpose, observe that $q_{0}=q_{0} \backslash q_{0}$ is equivalent to $q_{0}=\emptyset, x \subseteq y$ is equivalent to $x \backslash y=\emptyset$, and $x=y \cap z$ is equivalent to $x=y \backslash(y \backslash z)$. Therefore, the literal $x \neq t$, where $x$ is a variable and $t$ denotes one of the terms $y \cup z, y \backslash z$, or $D f$, plainly equisatisfiable to $u \in w \wedge w \subseteq(x \backslash t) \cup(t \backslash x)$, with $u$ and $w$ newly introduced variables, is equisatisfiable to a conjunction of positive atoms of the form (1). Also, $x \notin y$ is equisatisfiable to $x \in w \wedge w \cap y=\emptyset$. Likewise, $\neg \operatorname{PAIR} \_\mathrm{IN}(x, y, f)$ is equisatisfiable to $\operatorname{PAIR} I N(x, y, g) \wedge g \cap f=\emptyset$. Moreover, $\neg \operatorname{INV}(f, g)$ is equisatisfiable to (PAIR_IN $(x, y, f) \wedge \neg \operatorname{PAIR} \operatorname{IN}(y, x, g)) \vee(\neg \operatorname{PAIR} \operatorname{IN}(x, y, f) \wedge$ PAIR IN $(y, x, g))$. Finally, it is plain that $\neg \operatorname{SINGLEVALUED~}(f)$ is equisatisfiable to PAIR IN $(x, y, f) \wedge \operatorname{PAIR} \_\mathrm{IN}\left(x, y^{\prime}, f\right) \wedge y \neq y^{\prime}$, which is easily expressible as a conjunction of positive atoms of type (1). It is convenient to restrict ourselves, without loss of generality, to the problem of injective satisfiability, where a set-theoretic formula $P$ is said to be injectively satisfied by a model $M$ if $M$ satisfies $P$ and $M$ maps distinct variables into distinct sets (in which case $M$ is called an injective model of $P$ ). Plainly, as shown in [Can], the injective satisfiability problem is equivalent to the ordinary satisfiability problem.

Summing up we have

LEMMA 1.1 The satisfiability problem for propositional combinations of atoms of type (1) is equivalent to the injective satisfiability problem for conjunctions of positive atoms of type (1).

In the above discussion, we showed that constructs $q_{0}=\emptyset, x \subseteq y, x=y \cap z$ (and their negations) can be expressed in the language $\mathcal{L}$. Other constructs expressible in $\mathcal{L}$ are:

$r=R f$, where $R f=\{q:[p, q] \in \operatorname{pairs}(f)$, for some $p\}$;

$R \operatorname{ESTR}(g, f, x)$, standing for $(\forall p)(\forall q)([p, q] \in g \leftrightarrow([p, q] \in f \wedge p \in x))$;

$y=f[x]$, where $f[x]=\{q:[p, q] \in \operatorname{pairs}(f)$, for some $p$ in $x\}$

$y=f^{-1}[x]$, where $f^{-1}[x]=\{p:[p, q] \in$ pairs $(f)$, for some $q$ in $x\}$;

$\operatorname{INJECTIVE}(f)$, standing for $(\forall p)\left(\forall p^{\prime}\right)(\forall q)\left([p, q] \in f \wedge\left[p^{\prime}, q\right] \in f \rightarrow p=p^{\prime}\right)$.

Indeed, a clause of type $r=R f$ can be eliminated by $r=D g \wedge \operatorname{INV}(g, f)$, with $g$ a new variable; $\operatorname{RESTR}(g, f, x)$ is equivalent to $D(f \backslash g) \cap x=\emptyset \wedge$ $D(g \backslash f)=\emptyset \wedge D g \subseteq x ; y=f[x]$ is equisatisfiable to $\operatorname{RESTR}(g, f, x) \wedge y=$ 
$R g ; y=f^{-1}[x]$ is equisatisfiable to $\operatorname{INV}(g, f) \wedge y=g[x] ; \operatorname{INJECTIVE}(f)$ is equisatisfiable to $\operatorname{INV}(g, f) \wedge \operatorname{SINGLEVALUED}(g)$ whereas clauses of type $\neg \operatorname{INJECTIVE}(f)$ can be eliminated by $\operatorname{INV}(g, f) \wedge \neg \operatorname{SINGLEVALUED}(g)$.

Before closing the present section, we intend to introduce some terminology on graphs which will be used later on in Section 3 (cf. [Jec] and [PaP]).

By a graph $G$ we mean a set $N$ of nodes together with a set $E \subseteq N \times N$ of edges. Usually we write $u \Rightarrow v$ to denote the edge which connects the startpoint $u$ with the endpoint $v$.

DEFINITION 1.2 A graph $G=(N, E)$ is said to be well-founded if it has no infinite descending chain.

Notice that an analogue of the arithmetic induction principle holds for wellfounded graphs. As an application, given a well-founded graph $G=(N, E)$, a notion of height can be easily defined on $N$. We put inductively

$$
\begin{gathered}
\text { height }(v)=0 \text { if } v \text { has no predecessors, } \\
\operatorname{height}(v)=\sup \{\operatorname{height}(u)+1: u \Rightarrow v \text { is in } E\} .
\end{gathered}
$$

DEFINITION 1.3 A graph $G=(N, E)$ is said to be (quasi-)extensional, if for all $v_{1}, v_{2} \in N$,

$$
\left\{u \in N: u \Rightarrow v_{1} \text { is in } E\right\}=\left\{u \in N: u \Rightarrow v_{2} \text { is in } E\right\} \quad(\neq \emptyset)
$$

implies $v_{1}=v_{2}$.

Finally we will need the concept of representation of a well-founded graph.

DEFINITION 1.4 A function $\mathrm{R}$ defined on the set $\mathrm{N}$ of nodes of a wellfounded graph $\mathrm{G}=(\mathrm{N}, \mathrm{E})$ and with values on a class of sets is called a representation of $\mathrm{G}$ if for all $v_{1}, v_{2} \in N$

(i) $R\left(v_{1}\right)=R\left(v_{2}\right)$ implies $v_{1}=v_{2}$,

(ii) $R\left(v_{1}\right) \in R\left(v_{2}\right)$ if and only if $v_{1} \Rightarrow v_{2}$ is in $E$.

Let $G=(N, E)$ be a well-founded graph and let $I$ be a function defined on $N$ and with values on a class of sets. Put inductively $R(v)=\{R(u): u \Rightarrow$ $v$ is in $E\} \cup I(v) .[\mathrm{PaP}]$ gives necessary and sufficient conditions on $I$ for $R$ to be a representation of $G$. 


\section{THE DECISION ALGORITHM}

Let $P$ be a conjunction of simple clauses of type (1). For technical reasons, and without any loss of generality, we assume that $P$ contains the following clauses

$$
q_{0}=q_{0} \backslash q_{0}, U=D U, \operatorname{INV}(U, U), x \backslash U=q_{0}, x \in U, \operatorname{PAIR} \operatorname{IN}(x, y, U),
$$

for all variables $x$ and $y$ occurring in $P$ and distinct from $U$, where the variable $U$ occurs in $P$ only within the clauses (3). To prove that the above assumption does not affect in any way our decidability result, we only need to verify that given a set $Q$ of clauses of type (1), and putting

$$
\begin{aligned}
Q^{\prime} \equiv Q & \wedge q_{0}=q_{0} \backslash q_{0} \wedge U=D U \wedge \operatorname{INV}(U, U) \\
& \wedge \bigwedge_{\substack{x, y \text { occur } \\
\text { in } Q}}\left(x \backslash U=q_{0} \wedge x \in U \wedge \text { PAIR_IN }(x, y, U)\right)
\end{aligned}
$$

with $q_{0}$ and $U$ new variables not already occurring in $Q$, then $Q$ and $Q^{\prime}$ are equisatisfiable.

Clearly any model $M^{\prime}$ of $Q^{\prime}$ is a model for $Q$. On the other hand let $M$ be a model for $Q$ and show how $M$ can be extended to a model for $Q^{\prime}$. Firstly', put $M q_{0}=\emptyset$, so that $q_{0}=q_{0} \backslash q_{0}$ is true in the model $M$ so extended. Next let $\alpha$ be a limit ordinal such that

$$
\operatorname{rank}(M x)<\alpha \text {, for all } x \text { occurring in } Q .
$$

(See [Jec] or [Can] for the definitions of limit ordinal, rank, von Neumann hierarchy of sets, etc.). Then we put

$$
M U=\mathcal{V}_{\alpha}
$$

where $\mathcal{V}_{\alpha}$ is the $\alpha$-th level of the von Neumann hierarchy of all sets, i.e. $\mathcal{V}_{\alpha}$ is the set of all sets having rank less than the ordinal $\alpha$. It is immediate to verify that $M$ so extended models correctly all conjuncts in $Q^{\prime}$.

So, let $P$ be a conjunction of literals of type (1) which satisfies the additional hypothesis introduced at the beginning of the present section. Let $M$ be an injective model of $P$. Below we will derive a collection of effectively verifiable conditions on the structure of $P$ which are necessary for $P$ to be injectively satisfiable. In the next section we will prove that such conditions are also sufficient for the injective satisfiability of $P$, therefore proving, in view of Lemma 1.1 , that the class of formulae in the language $\mathcal{L}$ has a solvable satisfiability problem. 

of $P$.

Up to the end of this section $M$ will denote a given and fixed injective model

Let $V=\left\{y_{1}, y_{2}, \ldots, y_{m}\right\}$ be the collection of all distinct variables occurring in $P$.

The presence in $P$ of clauses (3) yields the following lemma.

LEMMA 2.1 (a) $M x \in U,[M x, M y] \in M U$, and $M x \subseteq M U$, for all variables $x, y$ in $V \backslash\{U\}$.

(b) If $[p, q] \in M U$ then $p \in M U$. Conversely, if $p \in M U$, then $[p, q] \in M U$, for some $q$.

(c) $[p, q] \in M U$ if and only if $[q, p] \in M U$, for all pairs $[p, q]$.

Let $\sigma_{1}, \sigma_{2}, \ldots, \sigma_{n}$ be the nonempty regions of the Venn diagram of the sets $M y_{1}, M y_{2}, \ldots, M y_{m}$ in the universe $M U$ (notice that by Lemma 2.1(a) $\left.\bigcup_{i=1}^{m} M y_{i}=M U\right)$. Then each set $\sigma_{i}$ is either wholly contained in $M x$ or wholly disjoint from it, for each $x$ in $V$, and we can define

$$
\pi_{i}(y)=\left\{\begin{array}{ll}
0 & \text { if } \sigma_{i} \cap M y=\emptyset \\
1 & \text { if } \sigma_{i} \subseteq M y
\end{array},\right.
$$

where $y$ ranges over $V$.

We put $\Pi=\left\{\pi_{1}, \pi_{2} \ldots, \pi_{n}\right\}$. Notice that $\Pi$ is a subset of the set of all $0 / 1$-valued functions defined on $V$, which is easily calculated. Furthermore, for each $y$ in $V$ we have

$$
M y=\bigcup_{\substack{\pi \in \Pi \\ \wedge(y)=1}} \sigma^{\pi}
$$

where we designate by $\sigma^{\pi}$ the region of the Ven diagram relative to the model $M$ and the set of clauses $P$ which induces the function $\pi$, according to the definition (4).

From (5) and the disjointness of the sets $\sigma$ 's, we can easily deduce some properties of the maps $\pi$ 's. Let $x=y \cup z$ occur in $P$. Then $M x=M y \cup M z$, i.e.

$$
\bigcup_{\pi(x)=1} \sigma^{\pi}=\bigcup_{\pi(y)=1} \sigma^{\pi} \cup \bigcup_{\pi(z)=1} \sigma^{\pi}=\bigcup_{\pi(y)=1 \vee \pi(z)=1} \sigma^{\pi} .
$$

Hence for each $\pi \in \Pi, \pi(x)=\pi(y) \vee \pi(z)$, where we have identified 0 and 1 with the truth values "false" and "true", respectively. Analogously, if $x=y \backslash z$ occurs in $P$, then we can prove that $\pi(x)=\pi(y) \wedge \neg \pi(z)$, for all $\pi$ in $\Pi$. 
DEFINITION 2.2 A $0 / 1$-valued function $\pi$ over $\mathrm{V}$ such that $\pi(x)=\pi(y) \mathrm{V}$ $\pi(z)$ (resp. $\pi(x)=\pi(y) \wedge \neg \pi(z)$ ) whenever $x=y \cup z$ (resp. $x=y \backslash z$ ) occurs in $P$ is called a place of $\mathrm{P}$.

We have therefore showed that $\Pi$ is a set of places of $P$.

Remark. In what follows we will often write $\pi \subseteq x$ when $\pi(x)=1$.

Next, let $x$ be in $V \backslash\{U\}$. It follows from Lemma 2.1(a) that $M x \in M U$ and therefore $M x \in \sigma^{\pi^{x}}$, for some place $\pi^{x}$. Notice that if $x \in y$ occurs in $P$, then $M x \in M y$, which in particular implies $\sigma^{\pi^{x}} \subseteq M y$, i.e. $\pi^{x}(y)=1$.

DEFINITION 2.3 Given a variable $\mathrm{x}$ in $V \backslash\{U\}$, a place $\pi$ is said to be at the variable $\mathrm{x}$ if $\pi(y)=1$ for all $\in$-clauses $x \in y$ appearing in $P$.

Hence we have shown that the map $x \mapsto \pi^{x}$ defined above associates each variable in $P$ distinct from $U$ with a place at the same variable. Analogously, we can set the following definition.

DEFINITION 2.4 Given two variables $\mathrm{x}$ and $\mathrm{y}$ in $V \backslash\{U\}$, a place $\pi$ is said to be at the pair $[x, y]$ if $\pi(f)=1$ for all clauses of type PAIR_IN(x,y,f) occurring in $\mathrm{P}$.

Therefore, for every $x, y$ in $V \backslash\{U\}$, by letting $\pi^{x, y}$ be the place of $P$ such that $[M x, M y] \in \sigma^{\pi^{x, y}}$ (cf. Lemma 2.1(a)), we have that $\pi^{x, y}$ is a place of $P$ at the pair $[x, y]$.

Having proved the existence of the set of places $\Pi$ and of the maps $x \mapsto \pi^{x}$ and $(x, y) \mapsto \pi^{x, y}$, such that $\pi^{x}$ and $\pi^{x, y}$ are places of $P$ respectively at the variable $x$ and at the pair $[x, y]$, we begin by listing a collection of conditions which are necessary for $P$ to be satisfiable.

Let $x$ and $y$ be any two distinct variables in $P$. Then, as the model $M$ is injective, $M x \neq M y$, so that by (5) there must exist a place $\pi \in \Pi$ such that $\pi(x) \neq \pi(y)$. This gives us a first necessary condition.

Condition C1. For all distinct $x, y$ occurring in $P$, there exists a place of $P$, $\pi \in \Pi$ such that $\pi(x) \neq \pi(y)$.

Next we observe that the rank ordering over $\left\{\sigma_{1}, \sigma_{2}, \ldots, \sigma_{n}\right\}$ ind uces a linear ordering < over $\Pi$ such that

$$
\text { if } \operatorname{rank}\left(\sigma^{\alpha}\right)<\operatorname{rank}\left(\sigma^{\beta}\right) \text { then } \alpha<\beta \text {, for all } \alpha, \beta \in \Pi \text {. }
$$


Let $\pi(x)=1$, for some $\pi \in \Pi$ and $x \in V \backslash\{U\}$. Then, plainly, $\operatorname{rank}\left(\sigma^{\pi}\right) \leq$ $\operatorname{rank}(M x)<\operatorname{rank}\left(\sigma^{\pi^{x}}\right)$, so that $\pi<\pi^{x}$. This yields a second necessary condition.

Condition C2. If $\pi(x)=1$, with $\pi \in$ II and $x \in V \backslash\{U\}$, then $\pi<\pi^{x}$.

It is easy to slow that in the absence of the map constructs $D$, INV, PAIR_IN, and SINGLEVALUED, conditions $\mathrm{C} 1$ and $\mathrm{C} 2$ are also sufficient for the injective satisfiability of $P$ (see [FOS]; in fact [FOS] gives an effective procedure which produces a model of $P$, when one such exists).

All the essential complications that need to be faced are connected with the presence in $P$ of finitely many clauses of the form $d=D f, \operatorname{INV}(f, g)$, PAIR IN $(x, y, f)$, SINGLEVALUED $(f)$. Note, for example, that in the presence of such clauses a satisfiable set of statements may possess infinite models only, the statement

$$
f \neq \emptyset \wedge f=D f
$$

being a case in point. This has the model

$$
f=\{0,[0,1],[[0,1], 2],[[[0,1], 2], 3], \ldots\},
$$

but since each $x \in f$ is a member of a member of a member of some $y \in f,(7)$ cannot have finite models.

The following definitions take a step toward elucidating the logical weight of clauses in $P$ of type $d=D f, \operatorname{INV}(f, g)$, and SINGLEVALUED $(f)$.

For eaclı $\alpha \in$ II, put

$$
\begin{gathered}
\operatorname{dom}(\alpha)=\left\{\beta \in \Pi: \operatorname{Dom}\left(\sigma^{\alpha}\right) \cap \sigma^{\beta} \neq \emptyset\right\}, \\
\operatorname{inv}(\alpha)=\left\{\beta \in \Pi: \text { there exists }[p, q] \in \sigma^{\alpha} \text { such that }[q, p] \in \sigma^{\beta}\right\},
\end{gathered}
$$

so that $d o m$ and inv are both maps from $\Pi$ into pow(I).

The maps dom and inv have some useful properties.

Let $\beta \in \operatorname{dom}(\alpha)$ and let $d=D f$ be a $D$-clause of $P$ such that $\alpha \subseteq f$. Hence $\sigma^{\alpha} \subseteq M f$, so that $\operatorname{Dom}\left(\sigma^{\alpha}\right) \subseteq \operatorname{Dom}(M f)=M d$, which by (8) yields $\sigma^{\beta} \subseteq M d$, i.e. $\beta \subseteq d$.

Next, let $\beta \in \operatorname{inv}(\alpha)$. It follows immediately from the definition (9) itself that $\alpha \in \operatorname{inv}(\beta)$. Moreover, assume that either $\operatorname{INV}(f, g)$ or $\operatorname{INV}(g, f)$ occurs in $P$. If $\alpha \subseteq f$, i.e. $\sigma^{\alpha} \subseteq M f$, let $[p, q] \in \sigma^{\alpha}$ such that $[q, p] \in \sigma^{\beta}$. As $[p, q] \in M f$, we have $[q, p] \in M g$ implying $\sigma^{\beta} \subseteq M g$, i.e. $\beta \subseteq g$. Analogously, we can prove that if $\beta \subseteq g$ then $\alpha \subseteq f$; therefore, summing up, we have proved that if 
$\beta \in$ inv $\alpha$ and either $\operatorname{INV}(f, g)$ or $\operatorname{INV}(g, f)$ occurs in $P$, then $\alpha(f)=1$ if and only if $\beta(g)=1$.

Notice also that if $\beta \in \operatorname{inv}(\alpha)$, then pairs $\left(\sigma^{\alpha}\right)$, pairs $\left(\sigma^{\beta}\right) \neq \emptyset$, i.e. $\operatorname{Dom}\left(\sigma^{\alpha}\right)$, $\operatorname{Dom}\left(\sigma^{\beta}\right) \neq \emptyset$, so that by Lemma 2.1 we have $\operatorname{Dom}\left(\sigma^{\alpha}\right), \operatorname{Dom}\left(\sigma^{\beta}\right) \subseteq M U$, which in turn implies $\operatorname{dom}(\alpha), \operatorname{dom}(\beta) \neq \emptyset$. In addition, if $\operatorname{dom}(\alpha) \neq \emptyset$, then, by (8), pairs $\left(\sigma^{\alpha}\right) \neq \emptyset$. But, from Lemma $2.1(\mathrm{c}),\left(\operatorname{pairs}\left(\sigma^{\alpha}\right)\right)^{-1} \subseteq M U$, thereby proving that $\operatorname{inv}(\alpha) \neq \emptyset$.

The preceding discussion gives us the following necessary conditions.

Condition C3. If $\beta \in \operatorname{dom}(\alpha)$, then

(i) inv $(\alpha) \neq \emptyset$ :

(ii) for all $D$-clauses $d=D f$ in $P$, if $a(f)=1$ then $\beta(d)=1$.

Condition C4. If $\beta \in \operatorname{inv}(\alpha)$, then

(i) $\alpha \in \operatorname{inv}(\beta)$;

(ii) $\operatorname{dom}(\alpha) \neq \emptyset$;

(iii) for all INV-clauses $\operatorname{INV}(f, g)$ or $\operatorname{INV}(g, f)$ in $P, \alpha(f)=1$ if and only if $\beta(g)=1$.

In order to state the next condition, we need to introduce the notion of $D$-pair.

DEFINITION $2.5 A D$-pair (relative to $P, \Pi$, dom, etc.) is a pair $(\Gamma, \pi)$ with $\Gamma \subseteq \Pi$ and $\pi \in \Pi$ such that

(i) $\Gamma \neq \emptyset$;

(ii) $\pi \in \operatorname{dom}(\gamma)$, for all $\gamma \in \Gamma$;

(iii) for all $\gamma_{1}, \gamma_{2} \in \Gamma$, if $\gamma_{1}(f)=\gamma_{2}(f)=1$, for some variable $f$ such that the clause SINGLEVALUED $(f)$ appears in $P$, then $\gamma_{1}=\gamma_{2}$;

(iv) for all D-clauses $d=D f$ present in $P$, if $\pi(d)=1$ then there exists $\gamma_{0} \in \Gamma$ such that $\gamma_{0}(f)=1$.

We have the following lemma. 
LEMMA 2.6 Let $\pi \in \Pi$ and let $p \in \sigma^{\pi}$. Put

$$
\Gamma_{p}=\left\{\gamma \in \Pi: p \in \operatorname{Dom}\left(\sigma^{\gamma}\right)\right\} .
$$

Then $\left(\Gamma_{p}, \pi\right)$ is a D-pair.

Proof. We need to verify that conditions (i)-(iv) of Definition 2.5 are all satisfied.

Condition (i) follows immediately from Lemma 2.1(b).

Concerning (ii), note that if $\gamma \in \Gamma_{p}$ then $p \in \operatorname{Dom}\left(\sigma^{\gamma}\right) \cap \sigma^{\pi}$, i.e., by (8), $\pi \in \operatorname{dom}(\gamma)$.

Next let $\gamma_{1}, \gamma_{2} \in \Gamma$ and suppose that $\gamma_{1}(f)=\gamma_{2}(f)=1$, where SINGLEVALUED $(f)$ occurs in $P$. We have $p \in \operatorname{Dom}\left(\sigma^{\gamma_{1}}\right) \cap \operatorname{Dom}\left(\sigma^{\gamma_{2}}\right)$, that is there exist $q_{1}$ and $q_{2}$ such that $\left[p, q_{1}\right] \in \sigma^{\gamma_{1}}$ and $\left[p, q_{2}\right] \in \sigma^{\gamma_{2}}$. But $\sigma^{\gamma_{1}} \cup \sigma^{\gamma_{2}} \subseteq M f$ and $M f$ is singlevalued. Therefore $q_{1}$ must coincide with $q_{2}$, i.e. $\left[p, q_{1}\right] \in \sigma^{\gamma_{1}} \cap \sigma^{\gamma_{2}}$. As the sets $\sigma$ 's are pairwise disjoint, it follows that $\gamma_{1}=\gamma_{2}$, thus verifying condition (iii).

Finally, as regards (iv), let $d=D f$ be a $D$-clause in $P$ such that $\pi(d)=1$. Then $p \in \sigma^{\pi} \subseteq M d=\operatorname{Dom}(M f)$, i.e. $[p, q] \in M f=\bigcup_{\gamma(f)=1} \sigma^{\gamma}$, for some $q$, i.e. $[p, q] \in \sigma^{\gamma_{0}}$ for some place $\gamma_{0}$ such that $\gamma_{0}(f)=1$. In particular one has $p \in \operatorname{Dom}\left(\sigma^{\gamma_{0}}\right) \cap \sigma^{\pi}$, which yields $\gamma_{0} \in \Gamma$ and in turn completes the verification of condition (iv).

Let $\alpha \in \Pi$ be such that $\operatorname{dom}(\alpha) \neq \emptyset$. Hence there must exist $\pi \in \Pi$ such that $\operatorname{Dom}\left(\sigma^{\alpha}\right) \cap \sigma^{\pi} \neq \emptyset$. Let $p \in \operatorname{Dom}\left(\sigma^{\alpha}\right) \cap \sigma^{\pi}$. From the preceding lemma it follows that $\left(\Gamma_{p}, \pi\right)$ is a $D$-pair, and since obviously $\alpha \in \Gamma_{p}$, we have the following necessary condition.

Condition C5. Let $\alpha \in \Pi$ II such that $\operatorname{dom}(\alpha) \neq \emptyset$. Then there exists a D-pair $(\Gamma, \pi)$ such that $\alpha \in \Gamma$.

For any two sets $s, t$ write $s \in^{*} t$ if there exists a chain of intermediate elements $s_{1}, s_{2}, \ldots, s_{k}$, with $k \geq 0$, such that $s \in s_{1} \in s_{2} \in \cdots \in s_{k} \in t$. Then for each variable $x$ in $P$ distinct from $U$ we put

$$
\Pi_{x}=\left\{\pi \in \Pi: M x \epsilon^{*} \sigma^{\pi}\right\} .
$$

By definition, for every $x \in V \backslash\{U\}$ we have $M x \in \sigma^{\pi^{x}}$ (see the discussion preceding Definition 2.3). Hence we obtain another necessary condition.

Condition C6. For all $x$ in $V \backslash\{U\}, \pi^{x} \in \Pi_{x}$. 
Moreover, let $\alpha(x)=1$ and $\pi \in \Pi_{x}$, where $x \in V \backslash\{U\}$. Therefore $\sigma^{\alpha} \subseteq$ $M x \in^{*} \sigma^{\pi}$, which yields $\operatorname{rank}\left(\sigma^{\alpha}\right)<\operatorname{rank}\left(\sigma^{\pi}\right)$. By (6) the latter inequality implies $\alpha<\pi$. Hence we have

Condition C7. Let $\alpha(x)=1$ and $\pi \in \Pi_{x}$ for some $x \in V \backslash\{U\}$. Then $\alpha<\pi$.

Notice that condition $\mathrm{C} 2$ is a consequence of conditions $\mathrm{C} 6$ and $\mathrm{C} 7$.

Recall that, by definition, $[M x, M y] \in \sigma^{\pi^{x, y}}$, for all $x, y \in V \backslash\{U\}$ (see the discussion just after Definition 2.4). Therefore $\pi^{x, y} \in \Pi_{x} \cap \Pi_{y}$. Also, as $M x \in \operatorname{Dom}\left(\sigma^{\pi^{x, y}}\right) \cap \pi^{x}$, we have $\pi^{x} \in \operatorname{dom}\left(\pi^{x, y}\right)$. Finally, notice that $[M x, M y] \in \sigma^{\pi^{x, y}}$ and $[M y, M x] \in \sigma^{\pi^{y, x}}$, for all $x, y \in V \backslash\{U\}$. Hence (9) yields $\pi^{y, x} \in \operatorname{inv}\left(\pi^{x, y}\right)$.

Summing up the preceding discussion, we obtain the following condition which is necessary for the injective satisfiability of $P$.

Condition C8. For all $x, y \in I^{\prime} \backslash\{U\}$ we have:

(i) $\pi^{x, y} \in \Pi_{x} \cap \Pi_{y}$;

(ii) $\pi^{x} \in \operatorname{dom}\left(\pi^{x, y}\right)$;

(iii) $\pi^{y, x} \in \operatorname{inv}\left(\pi^{x, y}\right)$.

Put, as in (10), $\Gamma_{M x}=\left\{\gamma \in \Pi: M x \in \operatorname{Dom}\left(\sigma^{\gamma}\right)\right\}$, with $x \in V \backslash\{U\}$. Then clearly $\Gamma_{M_{x}} \subseteq \Pi_{x}$ and $\pi^{x, y} \in \Gamma_{M x}$, for each $y \in V \backslash\{U\}$. Moreover, for each $\gamma \in \Gamma_{M x}$, since $M x \in \operatorname{Dom}\left(\sigma^{\gamma}\right)$ then $[M x, q] \in \sigma^{\gamma}$ for some $q$. But therefore by Lemma $2.1(\mathrm{c})$ and $(9)[q, M x] \in \sigma^{\delta}$, for some $\delta \in$ inv $(\alpha)$. In particular, since $M x \epsilon^{*} \sigma^{\delta}$ we have also $\delta \in \operatorname{inv}(\alpha) \cap \Pi_{x}$. Observe, in addition, that since $M x \in \sigma^{\pi^{x}}$, from Lemma 2.6 it follows that $\left(\Gamma_{M x}, \pi^{x}\right)$ is a $D$-pair.

Hence we deduce the following necessary condition.

Condition C9. Let $x \in V \backslash\{U\}$. Then there exists a $D$-pair $\left(\Gamma, \pi^{x}\right)$ such that

(i) $\Gamma \subseteq \Pi_{x}$;

(ii) $\pi^{x, y} \in \Gamma$, for each $y$ in $V \backslash\{U\}$;

(iii) $\operatorname{inv}(\gamma) \cap \Pi_{x} \neq \emptyset$, for each $\gamma \in \Gamma$. 
Next, let $\pi \in \Pi$. Since $\sigma^{\pi} \subseteq M U=\operatorname{Dom}(M U)$, it follows that for some $\alpha \in \Pi \quad \sigma^{\pi} \cap \operatorname{Dom}\left(\sigma^{\alpha}\right) \neq \emptyset$. Therefore Lemma 2.6 implies that there exists a $D$-pair $(\Gamma, \pi)$.

If, moreover, $\pi \in \mathrm{II}_{x}$, for some $x \in V \backslash\{U\}$, then $M x \in^{*} \sigma^{\pi}$. Choose $p \in \sigma^{\pi}$ such that $M x \in \in^{*}\{p\}$. Then it follows again from Lemma 2.6 that $\left(\Gamma_{p}, \pi\right)$ is a $D$-pair. In addition, let $\gamma \in \Gamma_{p}$. We have $p \in \operatorname{Dom}\left(\sigma^{\gamma}\right)$, so that $M x \in^{*} \sigma^{\gamma}$, i.e. $\gamma \in \Pi_{x}$. Also, as $p \in \operatorname{Dom}\left(\sigma^{\gamma}\right),[p, q] \in \sigma^{\gamma}$ for some $q$. Lemma 2.1(c) implies that $[q, p] \in \sigma^{\delta}$ for some $\delta \in \Pi$. In fact, by (2.7), $\delta \in$ inv $(\gamma)$ and since $M x \in^{*} \sigma^{\delta}$, we have also $\delta \in \Pi_{x}$.

The preceding discussion is summarized in the following condition.

Condition C10. For each $\pi \in \mathrm{II}$ there exists a $D$-pair $(\Gamma, \pi)$. In addition, for each $\pi \in \Pi_{x}$, with $x \in V \backslash\{U\}$, there exists a D-pair $(\Gamma, \pi)$ such that $\Gamma \subseteq \Pi_{x}$ and, for each $\gamma \in \Gamma$, inv $(\gamma) \cap \Pi_{x} \neq \emptyset$.

Clauses of type SINGLEVALUED $(f)$ have already been taken into account within the definition of $D$-pairs. Another condition concerning such clauses, which also concludes our list of necessary conditions, is the following.

Condition C11. If the clause SINGLEVALUED $(f)$ appears in $f$, then for each $x, y, y^{\prime} \in V \backslash\{U\}$ such that $\pi^{x, y}(f)=\pi^{x, y^{\prime}}(f)=1$ we have $y=y^{\prime}$.

To show that condition $\mathrm{C} 11$ is necessary together with conditions $\mathrm{C} 1-\mathrm{C} 10$ for the injective satisfiability of $P$ we observe that if $\pi^{x, y}(f)=\pi^{x, y^{\prime}}(f)=1$, then $[M x, M y],\left[M x, M y^{\prime}\right] \in M f$. But $M f$ must be singlevalued, hence $M y=M y^{\prime}$. The equality $y=y^{\prime}$ follows immediately from the injectivity of $M$.

The results proved in this section can be summarized in the following lemma.

LEMMA 2.7 Let $P$ be a conjunction of simple clauses of type $x=y \cup z$, $x=y \backslash z, x \in y, d=D f, \operatorname{PAIR}$ IN $(x, y, f), \operatorname{INV}(f, g), \operatorname{SINGLEVALUED}(f)$, containing also clauses (3). If $P$ is injectively satisfiable, then there exist

(i) a set $\Pi$ of places of $P$;

(ii) a map $x \mapsto \pi^{x}$ from $V \backslash\{U\}$ into II such that $\pi^{x}$ is a place at the variable $x$, for all $x \in V \backslash\{U\}$;

(iii) a map $(x, y) \mapsto \pi^{x, y}$ from $(V \backslash\{U\})^{2}$ into $\Pi$ such that $\pi^{x, y}$ is a place at the pair $(x, y)$, for all $x, y \in V \backslash\{U\}$;

(iv) a linear ordering < over $\Pi$;

(v) two maps dom and inv from II into pow( $\Pi$ ); 
(vi) a map $x \mapsto \Pi_{x}$ from $V \backslash\{U\}$ into $\operatorname{pow}(\Pi) \backslash\{\emptyset\}$

such that conditions C1-C11 are satisfied.

Plainly, all conditions stated in the preceding lemma are effectively verifiable. Therefore, in order to establish our main result, to viz. that the class of unquantified set-theoretic formulae in the language $\mathcal{L}$ has a solvable decidability problem, it only remains to show that the conditions of Lemma 2.7 are also sufficient for the injective satisfiability of $P$.

This will be done in the next section.

\section{PROOF OF SUFFICIENCY OF CONDITIONS C1-C11}

Again, let $P$ be a conjunction of positive clauses of type (1) containing also clauses (3) and let $V$ be the set of variables occurring in $P$. Furthermore, assume that there exist $\Pi=\left\{\pi_{1}, \pi_{2}, \ldots, \pi_{n}\right\}, x \mapsto \pi^{x},[x, y] \mapsto \pi^{x, y},<$, dom: II $\rightarrow$ pow(I), inv : II $\rightarrow$ pow(II), $x \mapsto \Pi_{x}$ as from (i)-(vi) of Lemma 2.7 and satisfying conditions $\mathrm{C} 1-\mathrm{C} 11$ of the preceding section.

In this section we will show that under such hypotheses $P$ has an injective model. More specifically, we will prove that an infinite, well-founded, quasiextensional graph (called the skeleton model of $P$ ) can be built ( $\mathrm{cf}$. Definitions $1.2,1.3)$. The skeleton model of $P$ will satisfy certain closure properties which depend on $P$ and $\Pi, x \mapsto \pi^{x}, \ldots, x \mapsto \Pi_{x}$. Subsequently we will show that this graph admits a representation (cf. Definition 1.4) from which an injective model $M^{*}$ of $P$ can be easily extracted.

In the presentation of the below Construction Process (C.P.), we will make use of the following definition.

DEFINITION 3.1 For any two sets $\Gamma_{1}, \Gamma_{2} \subseteq \Pi$, we put

$\Gamma_{1} \prec \Gamma_{2}$ if there exist $\gamma_{2} \in \Gamma_{2}$ such that $\gamma_{1}<\gamma_{2}$, for all $\gamma_{1} \in \Gamma_{1}$.

In particular, for all $x, y \in V$, we put

$$
x \prec y \text { if }\{\pi \in \Pi: \pi \subseteq x\} \prec\{\pi \in \Pi: \pi \subseteq y\} .
$$

Notice that the relation $\prec$ is acyclic and therefore extendible to a linear ordering, which troughout the present section will be denoted by the same symbol $\prec$.

In detail, the skeleton model is constructed by the following infinite process. 


\section{CONSTRUCTION PROCESS}

\section{[INITIALIZATION PIIASE]}

(I.1) Let $v_{1}, v_{2}, v_{3}, \ldots$ be a countable infinity of distinct objects called nodes. Put

(I.2) $N \leftarrow \emptyset$

(I.3) $E \leftarrow \emptyset$

(I.4) $G \leftarrow(N, E)$,

where $G, N$ and $E$ stand respectively for the initial values of the skeleton model $G$, its set of nodes and edges.

\section{(I.5) FOR EACH $\Sigma \subseteq \Pi$ DO}

(I.6) pick an unused node $v_{\Sigma}$ and put

(I.7) $N \leftarrow N \cup\left\{v_{\Sigma}\right\}$

(I.8) node $(\Sigma)-v_{\Sigma}$.

\section{END FOR.}

[Note: The function "node" maps pow $(\Pi)$ into $N$. For simplicity, we will write node $(\pi)$ instead of node $(\{\pi\})$; also, for each variable $x$ occurring in $P$ we will write node $(x)$ in place of node $(\{\pi \in \Pi: \pi(x)=1\})$. $\}$

(I.9) Arrange the set of unusued nodes into $(n+2)$ infinite disjoint subsets $S_{1}, S_{2}, \ldots, S_{n}, S_{n+1}, S_{n+2}$, where $n=|\Pi|$.

[Comment: As will be clear from the code of the following FOR-loop, the nodes in $S_{i}$ will be made sons of node $\left(\pi_{i}\right)$, for $i=1,2, \ldots, n$. $S_{n+1}$ contains an infinite supply of nodes which will be used to force suitable closure properties. For technical reasons we will temporarily allow certain edges to be labeled. Nodes in $S_{n+2}$ will then be used in the elimination process of labeled edges.]

(I.10) FOR EACH $i \in\{1,2, \ldots, n\}$ DO

Put

(I.11) $N \leftarrow N \cup S_{i}$ 
(I.12) FOR EACH $v \in S_{3}$ DO

Put

(I.13) $E-E \cup\left\{v \Rightarrow \operatorname{node}\left(\pi_{i}\right)\right\}$

(1.14) Propagate $\left(v, \pi_{i}\right)$. (Note: The code for the procedure Propagate will be shown below.]

\section{END FOR EACH.}

\section{END FOR EACH.}

[Note: The purpose of the present algorithm is to force certain closure properties, which will be discussed at length below, on the skeleton model. To this end, each node $v$ in $N$ for which the edge $v \Rightarrow$ node $\left(\pi_{\imath}\right)$ is in $E$, for some $i=1,2, \ldots, n$, will opportunely be processed. It will therefore be convenient to arrange all nodes in $S_{1} \cup S_{2} \cup \cdots \cup S_{n}$ in an ordered list $L_{0}$. Also, we will maintain a second ordered list $L_{1}$ which will contain new nodes produced during the processing of old nodes. A "mark" function $m$ from nodes of $L_{0}$ and $L_{1}$ into subsets of $\Pi$ will also be maintained.]

(I.15) Arrange in any convenient way all nodes in $S_{1} \cup S_{2} \cup \cdots \cup S_{n}$ in an ordered list $L_{0}$.

(1.16) FOR EACH node $v$ in $L_{0}$ DO

Put

(1.17) $m(v)-\emptyset$

END FOR EACH.

(I.18) Initialize $L_{1}$ to the empty list.

(I.19) FOR EACH $x \in V \backslash\{U\}$ DO

Put

(I.20) $E-E \cup\left\{\operatorname{node}(x) \Rightarrow \operatorname{node}\left(\pi^{x}\right)\right\}$

(I.21) Add node $(x)$ at the end of the list $L_{1}$,

(I.22) Propagate $\left(\operatorname{node}(x), \operatorname{node}\left(\pi^{x}\right)\right)$.

Put

(1.23) $m(\operatorname{node}(x))=\left\{\pi^{x, y} \in \Pi: y \in V \backslash\{U\}\right\}$ 


\section{END FOR EACH.}

(I.24) FOR EACH $[x, y] \in(V \backslash\{U\})^{2}$ DO

(I.25) Pick a new node $v_{x, y}$ from $S_{n+1}$ and discard it from $S_{n+1}$.

[Comment: The node $v_{x, y}$ represents the pair $[x, y]$ ]

Put

(I.26) $N \leftarrow N \cup\left\{v_{x, y}\right\}$

(I.27) $E \leftarrow E \cup\left\{\operatorname{node}(x) \stackrel{L}{\Rightarrow} v_{x, y}, \operatorname{node}(y) \stackrel{R}{\Rightarrow} v_{x, y}, v_{x, y} \Rightarrow \operatorname{node}\left(\pi^{x, y}\right)\right\}$

(I.28) Add $v_{x, y}$ at the end of the list $L_{1}$.

(I.29) Propagate $\left(v_{x, y}, \operatorname{node}\left(\pi^{x, y}\right)\right)$

(I.30) $m\left(v_{x, y}\right)-\emptyset$

END FOR EACH.

[END INITIALIZATION PHASE]

[STABILIZATION PIIASE]

(S.1) $i-0$

(S.2) DO FOREVER

(S.3) $i-i+1$;

(S.4) $r \leftarrow i \bmod 2$;

(S.5) IF the list $L_{r}$ is empty THEN CONTINUE;

END IF.

(S.6) Let $v$ be the node contained in the first location of $L_{r}$. Discard $v$ from $L_{\tau}$.

(S.7) Let $\pi$ be the unique place in II such that $v \Rightarrow \operatorname{node}(\pi)$ is in $E$.

(S.8) Let $T_{v}=\{v\} \cup\{w \in N$ : there is a path leading from $w$ to $v\}$.

(S.9) CASE $T_{v} \cap\{\operatorname{node}(x): x \in V \backslash\{U\}\}=\emptyset$ :

(S.10) Let $(\Gamma, \pi)$ be a $D$-pair such that $m(v) \subseteq \Gamma$.

(S.11) FOR EACH $\gamma \in \Gamma \backslash m(v)$ DO

(S.12) Let $\iota_{\gamma} \in \operatorname{inv}(\gamma)$, and let $\left(I_{\gamma}, \beta_{\gamma}\right)$ be a $D$-pair such that $\iota_{\gamma} \in$ $I_{\gamma}$. 
(S.13) Stabilize $\left(v, \gamma, \iota_{\gamma}, \beta_{\gamma}\right)$ [Note: The code for the procedure Stabilize is shown below.]

END FOR EACH.

(S.14) CASE $T_{v} \cap\{\operatorname{node}(x): x \in V \backslash\{U\}\} \neq \emptyset$ :

(S.15) Let $x_{0}$ be a $\prec$-maximal variable such that node $\left(x_{0}\right) \in T_{v}$ (cf. Definition 3.1).

(S.16) Notice that $\pi \in \Pi_{I_{0}}$.

(S.17) Let $(\Gamma, \pi)$ be a $D$-pair such that $m(v) \subseteq \Gamma, \Gamma \subseteq \Pi_{x_{0}}$, and for each $\gamma \in \Gamma$ inv $(\gamma) \cap \Pi_{x_{0}} \neq \emptyset$.

(S.18) FOR EACH $\gamma \in \Gamma \backslash m(v)$ DO

(S.19) Let $\iota_{\gamma} \in \operatorname{inv}(\gamma) \cap \Pi_{x_{0}}$, and let $\left(I_{\gamma}, \beta_{\gamma}\right)$ be a $D$-pair such that $\iota_{\gamma} \in I_{\gamma}$.

(S.20) Stabilize $\left(v, \gamma, \iota_{,}, B_{\gamma}\right)$.

END FOR EACH.

END CASE.

END DO FOREVER.

\section{PROCEDURE Stabilize $(v, \lambda, \mu, v)$}

[Note: When Stabilize $(v, \lambda, \mu, \nu)$ is called, $v$ is a node in $N$ and $\lambda, \mu, \nu$ are places of $P$ in $\Pi$.]

(PS.1) Let $w$ be the first node in the list $L_{0}$ such that $m(w)=\emptyset$ and $w \Rightarrow \operatorname{node}(v)$ is in $E$.

(PS.2) Let $u_{v, w}$ and $u_{u^{\prime}, v}$ be two distinct nodes in the set $S_{n+1}$. Discard them from $S_{n+1}$.

Put

(PS.3) $N-N \cup\left\{u_{v, w}, u_{w, v}\right\}$;

(PS.4) $E \leftarrow E \cup\left\{v \stackrel{L}{\Rightarrow} u_{v, w}, w \stackrel{R}{\Rightarrow} u_{v, w}, w \stackrel{L}{\Rightarrow} u_{w, v}, v \stackrel{R}{\Rightarrow} u_{w, v}, u_{v, w} \Rightarrow \operatorname{node}(\lambda), u_{w, v} \Rightarrow\right.$ $\operatorname{node}(\mu)\}$.

(PS.5) Add $u_{v, w}$ and $u_{w, v}$ at the end of the list $L_{1}$.

(PS.6) Propagate $\left(u_{v, w}, \operatorname{node}(\lambda)\right)$; 


$$
\begin{aligned}
& \text { (PS.7) Propagate }\left(u_{w, v}, \operatorname{node}(\mu)\right) . \\
& (\text { PS.8) } m(w) \leftarrow\{\mu\} \\
& \left(\text { PS.9) } m\left(u_{v, w}\right) \leftarrow \emptyset\right. \\
& \left(\text { PS.10) } m\left(u_{w, v}\right)-\emptyset\right.
\end{aligned}
$$

END PROCEDURE Stabilize.

PROCEDURE Propagate $(v, \pi)$;

(PP.1) FOR EACH $\Sigma \subseteq \Pi$ such that $\{\pi\} \subseteq \Sigma$ DO

Put

(PP.2) $E \leftarrow E \cup\{v \Rightarrow \operatorname{node}(\Sigma)\}$

\section{END FOR EACH.}

END PROCEDURE Propagate.

[END STABILIZATION PHASE]

[COMPLETION PHASE]

(C.1) Arrange all startpoints of labeled edges contained in $E$ in an ordered list $L_{2}$ such that node $\left(q_{0}\right)$ is the first element in $L_{2}$.

(C.2) FOR EACH $v$ in $L_{2} \mathrm{DO}$

(C.3) Let $v^{\prime}, v_{L}$, and $v_{R}$ be distinct nodes in $S_{n+2}$. Discard them from $S_{n+2}$.

[Notice: In the following $\underline{0}$ and $\underline{2}$ will stand respectively for node $\left(q_{0}\right)$ and $\left.\left(\operatorname{node}\left(q_{0}\right)\right)_{L} \cdot\right]$

Put

(C.4) $N \leftarrow N \cup\left\{v^{\prime}, v_{L}, v_{R}\right\}$.

(C.5) $E \leftarrow E \cup\left\{v \Rightarrow v^{\prime}, v^{\prime} \Rightarrow v_{L}, \underline{0} \Rightarrow v_{L}, v^{\prime} \Rightarrow v_{R}, \underline{2} \Rightarrow v_{R}\right\}$.

\section{END FOR EACH.}

(C.6) FOR EACH labeled edge $v \stackrel{L}{\Rightarrow} w($ resp. $v \stackrel{R}{\Rightarrow} w)$ in $E$ DO 
(C.7) Substitute in $E$ the labeled edge $v \stackrel{L}{\Rightarrow} w$ (resp. $v \stackrel{R}{\Rightarrow} w$ ) with the unlabeled edge $v_{L} \Rightarrow w$ (resp. $v_{R} \Rightarrow w$ ).

END FOR EACH.

[END COMPLETION PHASE]

\section{END CONSTRUCTION PROCESS.}

Remark. In what follows, we will denote by $G_{I}=\left(N_{I}, E_{I}\right), G_{S}=\left(N_{S}, E_{S}\right)$, and $G_{C}=\left(N_{C}, E_{C}\right)$ the skeleton model as constructed at the end of the Initialization Phase, Stabilization Phase, and Completion Phase of the above C.P., respectively.

The above Construction Process contains some implicit assertions (cf. for example lines (S.7), (S.10), (S.12), (S.15), (S.16), (S.17), (S.19), (PS.1), etc.). Below we will show that under the hypotheses stated at the beginning of the present section, all assertions are true in any computation $K$ of the C.P. . Subsequently we will prove that the graph $G_{C}=\left(N_{C}, E_{C}\right)$ is well-founded and quasi-extensional (cf. Definitions 1.2 and 1.3). Also we will show that $G_{C}$ enjoys several closure properties which will allow us to define a suitable representation Repr of $G_{C}$ for which the assignment $M^{*} x=\operatorname{Repr}(\operatorname{node}(x))$, for each $x$ occurring in our conjunction $P$, is injective and satisfies $P$.

Let $K$ be a computation of the C.P. above.

We begin by stating some simple properties of the skeleton model $G_{C}$ which can be deduced by a mere inspection of the C.P. code.

LEMMA 3.2 (a) Let $v$ be a node such that $v \Rightarrow$ node $(\pi)$ is in $E_{C}$ for some $\pi \in \Pi$. Then, during the computation $K, v$ is inserted (and subsequently processed by Stabilize) either in $L_{0}$ or in $L_{1}$.

(b) Conversely, if during the computation $K$ a node is introduced in one of the lists $L_{0}$ and $L_{1}$, then there is a unique place $\pi \in \Pi$ such that the edge $v \Rightarrow \operatorname{node}(\pi)$ is in $E_{C}$ (for specificity, we will indicate this uniquely characterized place by ${ }^{v} \pi$ ).

(c) $N o u \in S_{1} \cup S_{2} \cup \cdots \cup S_{n}$ has any predecessors.

(d) node $\left(q_{0}\right)$ has no predecessors.

(e) For all $\Sigma \subseteq \Pi$ such that node $(\Sigma) \notin\{$ node $(x): x \in V \backslash\{U\}\}$, node $(\Sigma)$ has no outgoing edges. 
(f) After execution of (C.1), the elements of the list $L_{2}$ are exactly all nodes $v$ such that $v \Rightarrow \operatorname{node}(x)$ is in $E_{C}$ for some $\pi \in \Pi$.

Proof. Concerning (d), it is enough to notice that by (2.1) and Definition 2.2, $\left\{\pi \in \Pi\right.$ II $\left.\pi\left(q_{0}\right)=1\right\}=\emptyset$. The remaining points of the lemma can be easily proved by inspecting the code of the Construction Process.

Lemma 3.2(b) implies that assertion (S.7) is satisfied. By inducting on $K$ we will show that assertions (S.10), (S.12), (S.16), (S.17), and (S.19) are also satisfied.

Let $v$ be a node selected at line (S.6), let ${ }^{v} \pi$ be the unique place in $\Pi$ such that $v \Rightarrow \operatorname{node}\left({ }^{v} \pi\right)$ is in $E_{C}$ and assume that at the time (S.9) is executed, $T_{v} \cap\{\operatorname{node}(x): x \in V \backslash\{U\}\}=\emptyset$, i.e. $v$ has no ancestor, nor it is, of type node $(x)$, for all $x \in V \backslash\{U\}$. Since in particular $v \neq \operatorname{node}(x)$, for each $x \in$ $V \backslash\{U\}$, it follows that either $m(v)=\emptyset$, or the value $m(v)$ has been set during the execution of line (PS.8) within a call Stabilize $\left(v^{\prime}, \lambda, \mu, \nu\right)$. In the latter case, by induction, $\mu \in \operatorname{inv}(\lambda)$ and $\mu \in I_{\mu}$ for some $D$-pair $\left(I_{\mu}, \nu\right)$. Hence (PS.8) yields $m(v)=\{\mu\} \subseteq I_{\mu}$. Moreover, since by (PS.1) $\nu={ }^{v} \pi$, it follows that the $D$-pair $\left(I_{\mu}, \nu\right)$ satisfies all requirements in (S.10). If, on the other hand, $m(v)=\emptyset$, then the validity of (S.10) follows from condition C10.

So, let $\left(\Gamma,{ }^{v} \pi\right)$ be a $D$-pair such that $m(v) \subseteq \Gamma$ (as from (S.10)) and let $\gamma \in$ $\Gamma \backslash m(v)$ (as from (S.11)). Definition 2.5(ii) of a $D$-pair implies that $\operatorname{dom}(\gamma) \neq \emptyset$. Hence, conditions C3(i), C4(i) and (ii), and C5 yield that $\operatorname{inv}(\gamma) \neq \emptyset$, and that for each $\iota_{\gamma} \in \operatorname{inv}(\gamma)$ there exists a $D$-pair $\left(I_{\gamma}, \beta_{\gamma}\right)$ such that $\iota_{\gamma} \in I_{\gamma}$, thus completing the verification of assertion (S.12).

Next suppose that at the time $v$ was selected from $L_{0}$ or $L_{1}, T_{v} \cap$ $\{\operatorname{node}(x): x \in V \backslash\{U\}\} \neq \emptyset$. We will distinguish two cases, according to whether $v=\operatorname{node}(x)$, for some $x \in V \backslash\{U\}$, or not.

Case: $v=\operatorname{node}(x)$, with $x \in V \backslash\{U\}$. Let $x_{0}$ be the $\prec$-maximal variable such that $\operatorname{node}\left(x_{0}\right) \in T_{v}$. Then $x_{0} \equiv x$. In fact, let $u$ be an immediate predecessor of $v$. If $u \in S_{1} \cup S_{2} \cup \cdots \cup S_{n}$, then $T_{u} \cap\{\operatorname{node}(y): y \in V \backslash\{U\}\}=\emptyset$. On the other hand, if $u \in S_{n+1}$, then the edge connecting $u$ to $v$ was inserted into $E$ during the execution of a call Stabilize $\left(v^{\prime}, \lambda, \mu, \nu\right)$. Plainly, then

$$
T_{u} \cap\{\operatorname{node}(y): y \in V \backslash\{U\}\}=T_{v^{\prime}} \cap\{\operatorname{node}(y): y \in V \backslash\{U\}\} .
$$

It is then enough to show that if $\operatorname{node}(y) \in T_{u}$ for some $y \in V \backslash\{U\}$ and $u \Rightarrow v$ is in $E_{C}$, then $y \prec x$. Let $u \Rightarrow v$ and let $y_{0}$ be the $\prec$-maximal variable $\operatorname{such}$ that $\operatorname{node}\left(y_{0}\right) \in T_{u}$. From (12), node $\left(y_{0}\right) \in T_{v^{\prime}}$ and $y_{0}$ is also $\prec$-maximal among the variables $y$ such that $\operatorname{node}(y) \in T_{v^{\prime}}$. Therefore, by induction and 
by (S.16)-(S.19), it follow's that $\lambda, \mu \in \Pi_{y_{0}}$ and node $(x) \in\{\operatorname{node}(\lambda), \operatorname{node}(\mu)\}$. This in particular yields $|\{\alpha \in \Pi: \alpha(x)=1\}|=1$ and the unique place $\alpha$ such that $\alpha(x)=1$ is either $\lambda$ or $\mu$. If $\pi\left(y_{0}\right)=1$, then, by condition $\mathrm{C} 7, \pi<\lambda$ and $\pi<\mu$. Hence $y_{0} \prec x$ (cf. Definition 3.1), thereby showing that under the hypothesis that $v=\operatorname{node}(x)$, if node $(y) \in T_{v}$, for some $y \in V \backslash\{U\}$, then $y \preceq x$.

By $(1.20), \operatorname{node}(x) \Rightarrow \operatorname{node}\left(\pi^{x}\right)$ is in $E$. Therefore in the present case ${ }^{v} \pi=\pi^{x}$ which, by condition C6, is a member of $\Pi_{x}$. Thus assertion (S.16) is completely verified. Since $v=\operatorname{node}(x)$, line (I.23) of the Construction Process implies that $m(v)=\left\{\pi^{x, y} \in \Pi: y \in V \backslash\{U\}\right\}$. Condition C9 guarantees that there exists a $D$-pair $(\Gamma, \pi)$ such that $m(v) \subseteq \Gamma \subseteq \Pi_{x}$ and such that $\operatorname{inv}(\gamma) \cap \Pi_{x} \neq \emptyset$, for every $\gamma \in \Gamma$. Hence, plainly, assertions (S.17) and (S.19) hold, at least in the case in which $v=\operatorname{node}(x)$, for some $x \in V \backslash\{U\}$.

Case: $v \notin\{\operatorname{node}(x): x \in V \backslash\{U\}\}$. In this case the node $v$ has been inserted into $N$ during the execution of a call Stabilize $\left(v^{\prime}, \lambda, \mu, \nu\right)$, and since obviously $v$ has at least one predecessor, from (PS.1), (PS.5), (PS.9) and (PS.10) it follows that $m(v)=\emptyset$. Notice that since $v \neq \operatorname{node}(x)$, for all $x \in V \backslash\{U\}$, then $T_{v} \cap\{\operatorname{node}(x): x \in V \backslash\{U\}\}=T_{v^{\prime}} \cap\{\operatorname{node}(x): x \in V \backslash\{U\}\}$. Therefore, if $x_{0}$ is the $\prec$-maximal variable in $V \backslash\{U\}$ such that node $\left(x_{0}\right) \in T_{v}$, then as in the previous case, by inductive hypothesis we can prove that $\lambda, \mu \in \Pi_{x_{0}}$ and ${ }^{v} \pi \in\{\lambda, \mu\}$, i.e. (S.16) holds. Also, much as in the preceding case, it can be proved that assertions (S.17) and (S.19) are true.

Finally, we conclude the verification that all instructions in the Construction Process are executable by observing that at initialization the sets $S_{n+1}, S_{n+2}$ are infinite, and that for each $\pi \in \Pi$ the list $L_{0}$ contains infinitely many nodes $w$ such that $m(w)=\emptyset$ and $w \Rightarrow \operatorname{node}(\pi)$ is in $E_{C}$. Hence, in particular, instructions (I.25), (PS.1), (PS.2), (C.1) and (C.3) are always executable, when encountered.

The above discussion can be summarized in the following lemma.

LEMMA 3.3 Under the hypotheses stated at the beginning of the present section, each computation of the Construction Process is successful, in the sense that all assertions (resp. instructions) encountered are valid (resp. executable).

Next we prove that the graph $G_{C}=\left(N_{C}, E_{C}\right)$ is well-founded.

We will begin by proving that $G_{I}=\left(N_{I}, E_{I}\right)$ is well-founded. Observe that $N_{I}$ consists of $S_{1} \cup S_{2} \cup \cdots \cup S_{n}$ plus a finite number of additional nodes. Therefore, if $G_{I}$ were not well-founded, by Lemma 3.2(c) it would contain a finite cycle

$$
v_{0} \Rightarrow v_{1} \Rightarrow \cdots \Rightarrow v_{k-1} \Rightarrow v_{0}
$$


where the $v_{i}$ 's are eitler nodes of type node( $\left.\Sigma\right)$, with $\Sigma \subseteq \Pi$, or nodes of type $v_{x, y}$ introduced during the execution of the FOR-loop at (I.24). If $v_{i}$ is a node of type $v_{x, y}, i \in\{0,1, \ldots, k-1\}$, then by (I.27) $v_{i-1} \in\{\operatorname{node}(x)$, node $(y)\}$ and $v_{i+1}=\operatorname{node}\left(\Sigma_{x, y}\right)$, for some $\Sigma_{x, y} \subseteq \Pi$ such that $\pi^{x, y} \in \Sigma_{x, y}((i-1)$ and $(i+1)$ are to be taken modulo $k$ ). For specificity, suppose that $v_{i-1}=\operatorname{node}(x)=$ $\operatorname{node}(\{\pi \in \Pi: \pi(x)=1\})$. Then, from conditions C8(i) and C7 it follows that for each $\pi \subseteq x, \pi<\pi^{x, y}$. On the other hand, if $v_{i}$ is of type node $(x)$, for some $x \in V \backslash\{U\}$, then $v_{i+1}=\operatorname{node}\left(\Sigma_{x}\right)$, for some $\Sigma_{x} \subseteq \Pi$ such that $\pi^{x} \in \Sigma_{x}$. By C6, then, for every $\pi \subseteq x$ we have $\pi<\pi^{x}$. In any case we have shown that by deleting from the cycle $v_{0} \Rightarrow v_{1} \Rightarrow \cdots \Rightarrow v_{k-1} \Rightarrow v_{0}$ all nodes of type $v_{x, y}$, we obtain a subcycle of $\Rightarrow^{*}$ (i.e. the transitive closure of $\Rightarrow$ ), $v_{i_{0}} \Rightarrow^{*} v_{i_{1}} \Rightarrow^{*}$ $\cdots \Rightarrow^{*} v_{i_{k^{\prime}-1}} \Rightarrow^{*} v_{i_{0}}$, such that $v_{i j}=\operatorname{node}\left(\Sigma_{j}\right), j=0,1, \ldots, k^{\prime}-1$, and for each $j=0,1, \ldots, k^{\prime}-1$ there is an $\alpha_{j} \in \Sigma_{j}$ such that for all $\pi \in \Sigma_{j-1}$ we have $\pi<\alpha_{j}$. In particular we would have $\alpha_{0}<\alpha_{1}<\cdots<\alpha_{k^{\prime}-1}<\alpha_{0}$, which is a contradiction. Thus the graph $G_{I}=\left(N_{I}, E_{I}\right)$ is well-founded.

During the Stabilization Phase, new edges can be added to $G$ only by the procedure Stabilize. Notice that each call to Stabilize can cause only finitely many new edges to be added to $G$. Therefore to show that the Stabilization Phase preserves the well-foundedness of $G$, it is enough to prove that the acyclicity of $G$ cannot be disrupted by calls to the procedure Stabilize.

So assume that $G$ is acyclic prior to the execution of a call Stabilize $(v, \lambda, \mu, \nu)$. The effect on $G$ of the call Stabilize $(v, \lambda, \mu, \nu)$ is that two new nodes, $u_{v, w}$ and $u_{w, v}$, are added to $G$ together with the edges $v \stackrel{L}{\Rightarrow} u_{v, w}, w \stackrel{R}{\Rightarrow} u_{v, w}$, $v \stackrel{R}{\Rightarrow} u_{w, v}, w \stackrel{L}{\Rightarrow} u_{w, v}, u_{v, w} \Rightarrow \operatorname{node}\left(\Sigma_{\lambda}\right)$, for all $\Sigma_{\lambda} \subseteq \Pi$ such that $\lambda \in \Sigma_{\lambda}$, and $u_{w, v} \Rightarrow \operatorname{node}\left(\Sigma_{\mu}\right)$, for all $\Sigma_{\mu} \subseteq \Pi$ such that $\mu \in \Sigma_{\mu}$ (observe that $w$ is a node with no incoming edges and $w \Rightarrow \operatorname{node}(\nu)$ is in $E$; cf. (PS.1), Lemma $3.2(\mathrm{~b}),(\mathrm{c})$ and (I.15)). Plainly, the only way a cycle could be introduced into $G$ is that before the call Stabilize $(v, \lambda, \mu, \nu)$ is made, there was a path leading from node $\left(\Sigma^{*}\right)$ to $v$, for some $\Sigma^{*} \subseteq \Pi$ such that $\Sigma^{*} \cap\{\lambda, \mu\} \neq \emptyset$. But then, by Lemma $3.2(\mathrm{e}), \operatorname{node}\left(\Sigma^{*}\right)=\operatorname{node}\left(x^{*}\right)$, for some $x^{*} \in V \backslash\{U\}$. Hence we would have $T_{v} \cap\{\operatorname{node}(x): x \in V \backslash\{U\}\} \neq \emptyset$, thus precluding, by (S.9), the possibility that the call Stabilize $(v, \lambda, \mu, \nu)$ can be made from (S.13). But even the assumption that the call Stabilize $(v, \lambda, \mu, \nu)$ is made from (S.20) is contradictory. Indeed, by letting $x_{0}$ be the $\prec$-maximal variable such that node $\left(x_{0}\right) \in T_{v}$, from (S.15), (S.17), (S.19) it would follow that $\lambda, \mu \in \Pi_{x_{0}}$. Thus, by condition $\mathrm{C}$, we would have $\pi<\lambda$ and $\pi<\mu$, for each $\pi \subseteq x_{0}$, which, recalling that $\Sigma^{*} \cap\{\lambda, \mu\} \neq \emptyset$, in turn would imply $x_{0} \prec x^{*}$. This, plainly, contradicts the $\prec$-maximality of $x_{0}$, and consequently proves that no cycle can be introduced 
by the call Stabilize $(v, \lambda, \mu, \nu)$.

Hence, at the end of the Stabilization Phase the graph $G$ is still well-founded.

Finally, by observing that, essentially, the effect of the Completion Phase on $G$ is to substitute labeled edges $v \stackrel{L}{\Rightarrow} w$ (resp. $v \stackrel{R}{\Rightarrow} w$ ) by unlabeled paths $v \Rightarrow v^{\prime} \Rightarrow v_{L} \Rightarrow w$ (resp. $v \Rightarrow v^{\prime} \Rightarrow v_{R} \Rightarrow w$ ) of length 3 , it follows plainly that the well-foundedness of $G$ is also preserved during the Completion Phase.

Hence we have:

LEMMA 3.4 The graph $G_{C}=\left(N_{C}, E_{C}\right)$ produced by the computation $K$ is well-founded.

In order to prove that the graph $G_{C}=\left(N_{C}, I_{C}\right)$ is also quasi-extensional, we partition the set of nodes $N_{C}$ as follows. In view of Lemma 3.2(f), put

$C l_{1}=\left\{v^{\prime}: v \Rightarrow \operatorname{node}(\pi)\right.$, for some $\left.\pi \in \Pi\right\}$,

$C l_{2}=\left\{v_{L}: v \Rightarrow \operatorname{node}(\pi)\right.$, for some $\left.\pi \in \Pi\right\}$,

$C l_{3}=\left\{v_{R}: v \Rightarrow \operatorname{node}(\pi)\right.$, for some $\left.\pi \in \Pi\right\}$,

$C l_{4}=\{\operatorname{node}(\Sigma): \Sigma \subseteq \Pi\}$,

$C l_{5}=S_{\mathrm{I}} \cup S_{2} \cup \cdots \cup S_{n}$ (cf. (I.9)),

$C l_{6}=N_{C} \backslash \bigcup_{i=1}^{6} C l_{i}$

where $v^{\prime}, v_{L}, v_{R}$ have been introduced during the FOR-loop (C.2) of the Completion Phase.

The following lemma, which can proved by a simple inspection of the Construction Process code, lists some useful results concerning the above partition.

LEMMA 3.5 (a) The maps $v \mapsto v^{\prime}, v \mapsto v_{L}$, and $v \mapsto v_{R}$, from $\{v \in$ $N_{C}: v \Rightarrow \operatorname{node}(\pi)$, for some $\left.\Pi\right\}$ into $\mathrm{Cl}_{1}, \mathrm{Cl}_{2}$, and $\mathrm{Cl}_{3}$, respectively, defined during the FOR-loop (C.2) of the C.P. Completion Phase, are injective.

(b) For each $v^{\prime}$ in $\mathrm{Cl}_{1}, v$ is the unique immediate predessor of $v^{\prime}$.

(c) For each $v_{L}$ in $\mathrm{Cl}_{2}, v^{\prime}$ and $\underline{0}$ are the only immediate predecessor of $v_{L}$.

(d) For each $v_{R}$ in $\mathrm{Cl}_{3}, v^{\prime}$ and $\underline{2}$ are the only immediate predecessor of $v_{R}$.

(e) For each $\Sigma \subseteq \Pi$,

(e $\left.e_{1}\right)$ if $\Sigma=\emptyset$, then $\operatorname{node}(\Sigma)=\operatorname{node}\left(q_{0}\right)$ has no immediate predecessor;

$\left(e_{2}\right)$ if $\Sigma \neq \emptyset$, then node $(\Sigma)$ has infinitely many predecessors. 
(f) For each $u$ in $\mathrm{Cl}_{6}$ there exist two uniquely determined nodes $v$ and $w$ such that $v_{L}$ and $w_{R}$ are the only immediate predecessors of $u$. To stress on this dependence, we will index the node $u$ with the pair $v, w$ (thus agreeing with the notation used in (PS.2)).

(g) The partial map $(v, w) \mapsto u_{v, w}$ defined in (f) above is injective.

An immediate consequence of the preceding lemma is that for all $v_{1}, v_{2} \in$ $C l_{i}, i \neq 4$, if $\left\{u \in N_{C}: u \Rightarrow v_{1}\right.$ is in $\left.E_{C}\right\}=\left\{u \in N_{C}: u \Rightarrow v_{2}\right.$ is in $\left.E_{C}\right\} \neq \emptyset$, then $v_{1}=v_{2}$. On the other hand, if $v_{1}, v_{2} \in C l_{4}$, then $v_{1}=\operatorname{node}\left(\Sigma_{1}\right)$ and $v_{2}=\operatorname{node}\left(\Sigma_{2}\right)$ for some $\Sigma_{1}, \Sigma_{2} \subseteq \Pi$. If $v_{1} \neq v_{2}$, then $\Sigma_{1} \neq \Sigma_{2}$, so that there exist $\pi_{j} \in\left(\Sigma_{1} \backslash \Sigma_{2}\right) \cup\left(\Sigma_{2} \backslash \Sigma_{1}\right)$. Let $u^{*} \in S_{j}$. Therefore by inspecting the FORloop (I.10), we have that $u^{*} \Rightarrow v_{1}$ is in $E_{C}$ if and only if $u^{*} \Rightarrow v_{2}$ is not in $E_{C}$, thus showing that $\left\{u \in N_{C}: u \Rightarrow v_{1}\right.$ is in $\left.E_{C}\right\} \neq\left\{u \in N_{C}: u \Rightarrow v_{2}\right.$ is in $\left.E_{C}\right\}$.

To complete the proof of the quasi-extensionality of $G_{C}$, it only remains to show that if $v_{1} \in C l_{i}$ and $v_{2} \in C l_{j}$, with $i \neq j$ and $i, j \neq 5$, then there is a node $u$ such that the edge $u \Rightarrow v_{1}$ is in $E_{C}$ if and only if the edge $u \Rightarrow v_{2}$ is not in $E_{C}$. Since every node in $\mathrm{Cl}_{1}$ has only one immediate predecessor, every node in $\mathrm{Cl}_{2} \cup \mathrm{Cl}_{3} \cap \mathrm{Cl}_{6}$ has only two immediate predecessor, whereas every node in $C l_{\mathbf{4}} \backslash\left\{\operatorname{node}\left(q_{0}\right)\right\}$ has infinitely many immediate predecessor, we can further limit ourselves to verify the above property only for $i, j \in\{2,3,6\}$.

If $v_{1} \in C l_{2} \cap C l_{3}$ and $v_{2} \in C l_{6}$, then either $v_{1}=w_{L}$ or $v_{1}=w_{R}$, for some node $w$ such that the edge $w \Rightarrow \operatorname{node}(\pi)$ is in $E_{C}$, with $\pi \in \Pi$. But, in any case, by (b) and (c) of Lemma $3.5, w^{\prime} \Rightarrow v_{1}$ is in $E_{C}$, with $w^{\prime} \in C l_{1}$, whereas $w^{\prime} \Rightarrow v_{2}$ is not in $E_{C}$, since by (f) of the same lemma the immediate predecessors of $v_{2}$ are contained in $\mathrm{Cl}_{2} \cup \mathrm{Cl}_{3}$. On the other hand, let $v_{L} \in \mathrm{Cl}_{2}$ and $w_{R} \in \mathrm{Cl}_{3}$. Points (c) and (d) of Lemma 3.5 imply respectively that $\underline{0} \Rightarrow v_{L}$ and $\left\{u \in N_{C}: u \Rightarrow w_{R}\right.$ is in $\left.E_{C}\right\} \subseteq C l_{1} \cup C l_{2}$. Hence $\underline{0} \Rightarrow w_{R}$ is not in $E_{C}$, since $\underline{0} \in C l_{4}$. This concludes the proof that the graph $G_{C}$ is quasi-extensional. Thus we have:

LEMMA 3.6 At termination of the Construction Process, the graph $G_{C}=$ $\left(N_{C}, E_{C}\right)$ is quasi-extensional.

The following lemma collects some closure properties enjoyed by the graph $G_{C}$. 
LEMMA 3.7 (a) For each $x \in V \backslash\{U\}$, node $(x) \Rightarrow \operatorname{node}\left(\pi^{x}\right)$ is in $E_{C}$.

(b) For each $x, y \in V \backslash\{U\}$, there exists a unique node $u_{x, y} \in C_{6}$ such that $(\operatorname{node}(x))_{L} \Rightarrow u_{x, y},(\operatorname{node}(y))_{R} \Rightarrow u_{x, y}$, and $u_{x, y} \Rightarrow \operatorname{node}\left(\pi^{x, y}\right)$ are in $E_{C}$. (Note: $u_{x, y}$ is an abbreviation for $u_{\text {node( } x), \text { node(y); }}$ cf. Lemma 3.5(f).)

(c) Let $v$ be a node such that $v \Rightarrow \operatorname{node}\left({ }^{v} \pi\right)$ is in $E_{C}$, with ${ }^{v} \pi \in$ II. Then there exists a $D$-pair $\left(\Gamma,{ }^{v} \pi\right)$ such that for each $\gamma \in \Gamma$, there is a node $w$ for which $u_{v, w} \Rightarrow \operatorname{node}(\gamma)$ is in $E_{C}$, and viceversa, if for some node $w$ and place $\pi \in \Pi$ the edge $u_{v, w} \Rightarrow \operatorname{node}(\pi)$ is in $E_{C}$, then $\pi \in \Gamma$.

(d) Lei $u_{v, w_{1}} \Rightarrow \operatorname{node}(\pi), u_{v, w_{2}} \Rightarrow \operatorname{node}(\pi)$ be in $E_{C}$, for some $v, w_{1}, w_{2} \in N_{C}$, $\pi \in \Pi$ II such that $w_{1} \neq w_{2}$. Then $v, w_{1}, w_{2} \in\{\operatorname{node}(x): x \in V \backslash\{U\}\}$.

(e) If $u_{v, w} \Rightarrow \operatorname{node}(\alpha)$ is in $E_{C}$, for some $u_{v, w} \in C l_{6}$ and $\alpha \in \Pi$, then $u_{w, v} \in C l_{6}$ and for sonc $\beta \in \operatorname{inv}(\alpha) u_{w, v} \Rightarrow \operatorname{node}(\beta)$ is in $E_{C}$.

(f) For all $v \in N_{C}$ and $x \in V, v \Rightarrow \operatorname{node}(x)$ is in $E_{C}$ if and only if $v \Rightarrow$ $\operatorname{node}(\pi)$ is in $E_{C}$ for some $\pi \subseteq x$.

Proof. (a) and (b) follow immediately from (I.20) and (I.27), together with (C.5), respectively.

Concerning (c), let $v$ be a node in $N_{C}$ such that $v \Rightarrow \operatorname{node}\left({ }^{v} \pi\right)$ is in $E_{C}$. Lemma 3.2(a) implies that $v$ is put either in $L_{0}$ or in $L_{1}$ during the computation $K$. Hence eventually it will be selected during the Stabilization Phase at line (S.6). From (S.10), (S.11) and (S.17), (S.18), it follows that there is a $D$-pair $\left(\Gamma^{*},{ }^{v} \pi\right)$ such that, for each $\gamma \in \Gamma^{*} \backslash m(v), v_{L} \Rightarrow u_{v, w}$ and $u_{v, w} \Rightarrow \operatorname{node}(\gamma)$ are in $E_{C}$, for some node $w$. On the other hand, if $\gamma \in m(v)$, then the same conclusion follows either from (I.19) and (I.24) or from (PS.4), according to whether $v$ is of type node $(x), x \in V \backslash\{U\}$, or $v$ is the element selected at (PS.1) during the execution of a call to the procedure Stabilize.

Conversely, if for some node $w$ and some place $\pi^{*} \in \Pi$ the edge $u_{v, w} \Rightarrow$ $\operatorname{node}\left(\pi^{*}\right)$ is in $E_{C}$, then $v \stackrel{L}{\Rightarrow} u_{v, w}$ is in $E_{S}$. If $v \stackrel{L}{\Rightarrow} u_{v, w}$ is introduced into $E_{S}$ during the execution of (I.27), then $v=\operatorname{node}(x), w=\operatorname{node}(y)$, for some $x, y \in V \backslash\{U\}$, and $\pi^{*}=\pi^{x, y} \in m(\operatorname{node}(x))$ (cf. (I.23)). Therefore, by (S.17), $m(v) \subseteq \Gamma^{*}$, and in particular $\pi^{*} \in \Gamma^{*}$. On the other hand, if $v \stackrel{L}{\Rightarrow} u_{v, w}$ is introduced during the execution of a call Stabilize $\left(v, \pi^{*}, \mu, \nu\right)$, then by (S.10) and (S.17) $\pi^{*} \in \Gamma^{*}$. Finally, if $v \stackrel{L}{\Rightarrow} u_{v, w}$ is introduced during the execution of a call Stabilize $\left(w, \lambda, \pi^{*}, \nu\right)$, then $m(v)=\left\{\pi^{*}\right\}$, so that when eventually $v$ is selected at (S.6), (S.10) and (S.17) will imply that $\pi^{*} \in \Gamma^{*}$.

Concerning (d), let $v, w_{1}, w_{2} \in N_{C}, \pi \in \Pi$ be such that $w_{1} \neq w_{2}$ and $u_{v, w_{1}} \Rightarrow \operatorname{node}(\pi), u_{v, w_{2}} \Rightarrow \operatorname{node}(\pi)$ are in $E_{C}$. By (c) above there exists a 
$D$-pair $\left(\Gamma,{ }^{v} \pi\right)$ such that $\pi \in \Gamma$ and the edge $v \Rightarrow \operatorname{node}\left({ }^{v} \pi\right)$ is in $E_{C}$. If either $\pi \in \Gamma \backslash m(v)$ or $\pi \in m(v)$, where the value $m(v)$ was set at line (PS.8), then inspection of the FOR-loops (S.11) and (S.18), as well as of the procedure Stabilize, yields $w_{1}=w_{2}$, a contradiction. Therefore we must have $\pi \in m(v)$, where the value $m(v)$ was set at line (I.23), in which case inspection of the FOR-loop (I.24) yields the conclusion.

Next, as regards (e), let $u_{v, w} \Rightarrow \operatorname{node}(\alpha)$ be in $E_{C}$, for some $u_{v, w} \in C l_{6}$. If $u_{v, w}$ was introduced into $N_{C}$ at (I.26), then $v=\operatorname{node}(x)$ and $w=\operatorname{node}(y)$, for some $x, y \in V \backslash\{U\}$, and $\alpha=\pi^{x, y}$. Therefore, $u_{w, v} \Rightarrow \operatorname{node}\left(\pi^{y, x}\right)$ is introduced in $E_{C}$ during the execution of the FOR-loop (I.24), and it is enough to observe that by condition $\mathrm{C} 8$ (iii), $\pi^{y, x} \in \operatorname{inv}\left(\pi^{x, y}\right)=\operatorname{inv}(\alpha)$. On the other hand, if $u_{v, w}$ was introduced in to $N_{C}$ during the execution of a call Stabilize $\left(v_{1}, \lambda, \mu, \nu\right)$, with $\alpha \in\{\lambda, \mu\}$, then $u_{w, v}$ is also introduced during the same call and $u_{w, v} \Rightarrow$ $\operatorname{node}(\beta)$, where $\beta \in\{\lambda, \mu\} \backslash\{\alpha\}$. But from (S.12) and (S.19) it follows that $\mu \in \operatorname{inv}(\lambda)$, which by condition $\mathrm{C} 4(\mathrm{i})$ yields $\lambda \in \operatorname{inv}(\mu)$. Thus in any case $\beta \in \operatorname{inv}(\alpha)$, and the proof of (e) is completed.

Finally, concerning ( $f$ ) it is enough to observe that as soon as a new edge $v \Rightarrow \operatorname{node}(\pi)$ is introduced in $E_{C}$, the call Propagate $(v, \pi)$ is made, with the effect that edges $v \Rightarrow$ node $(\Sigma)$, for all $\Sigma \subseteq \Pi$ such that $\pi \in \Sigma$, are introduced into $E_{C}$. This completes the proof of the lemma.

Having proved that the graph $G_{C}$ is well-founded and quasi-extensional and that possesses the closure properties stated in the preceding lemma, we next define a suitable representation of $G_{C}$ from which subsequently an injective model of $P$ will be extracted.

Put

$$
A_{i}=\{\{3 i, 3 i+1,3 i+2\}\} \quad, \quad i=1,2, \ldots,
$$

and let $I$ be a biunivoque correspondence from $S_{1} \cup S_{2} \cup \cdots \cup S_{n}$ into $\left\{A_{i}: i=\right.$ $1,2, \ldots\}\left(S_{1} \cup S_{2} \cup \cdots \cup S_{n}\right.$ is the set of nodes in $N_{C} \backslash\left\{\operatorname{node}\left(q_{0}\right)\right\}$ with no immediate predecessors). Extend $I$ to the whole $N_{C}$ by putting $I(v)=\emptyset$, for all $v \in N_{C} \backslash\left(S_{1} \cup S_{2} \cup \cdots \cup S_{n}\right)$. Then by induction on height $(v)$, for all $v$ we put:

$$
\operatorname{Repr}(v)=\left\{\operatorname{Repr}(u): u \Rightarrow v \text { is in } E_{C}\right\} \cup I(v) .
$$

The map Repr is a representation of $G_{C}$ (in the sense of Definition 1.4), as proved in the following lemma. 
LEMMA 3.8 For all $u, v \in N_{C}$

(a) if $\operatorname{Repr}(u)=\operatorname{Repr}(v)$ then $u=v$;

(b) $\operatorname{Repr}(u) \in \operatorname{Repr}(v)$ if and only if $u \Rightarrow v$ is in $E_{C}$.

Proof. (a) We will proceed by induction on $M=\max ($ height $(u)$, height $(v)$ ). If $M=0$ then height $(u)=\operatorname{height}(v)=0$, i.e.

$$
u, v \in S_{1} \cup S_{2} \cup \cdots \cup S_{n} \cup\left\{\operatorname{node}\left(q_{0}\right)\right\} .
$$

Therefore by $(14) \operatorname{Repr}(u)=I(u)$ and $\operatorname{Repr}(v)=I(v)$. Thus by the injectivity of $I$ over $S_{1} \cup S_{2} \cup \cdots \cup S_{n} \cup\left\{\operatorname{node}\left(q_{0}\right)\right\}$, if $I(u)=I(v)$, then $u=v$. Concerning the inductive step, suppose that $\operatorname{Repr}(u)=\operatorname{Repr}(v)$. For specificity, assume that height $(u)>0$, so that $I(u)=\emptyset$. Let $w_{1} \Rightarrow u$ be in $E_{C}$ and let $t=\operatorname{Repr}\left(w_{1}\right)$. Notice that by Lemma 3.5, no node in $N_{C}$ has exactly three immediate predecessors. Therefore, by induction, $\left|\operatorname{Re} \operatorname{pr}\left(w_{1}\right)\right| \neq 3$, that is $\operatorname{Repr}\left(w_{1}\right) \in \operatorname{Repr}(v) \backslash I(v)$ (this in particular shows that $I(v)=\emptyset$ ). By (14) there must exist $w_{2} \in N_{C}$ such that $w_{2} \Rightarrow v$ is in $G_{C}$ and $\operatorname{Repr}\left(w_{2}\right)=\operatorname{Repr}\left(w_{1}\right)$. But max(height $\left(w_{1}\right)$, height $\left.\left(w_{2}\right)\right)<\max$ (height $(u)$, height $(v)$ ); hence by ind uction $w_{1}=w_{2}$, i.e. $w_{1} \Rightarrow v$ is in $G_{C}$. Likewise, we can prove that if $w_{1} \Rightarrow v$ is in $E_{C}$ then so is $w_{1} \Rightarrow u$. Thus by quasi-extensionality it follows $u=v$, completing the proof of (a).

(b) By definition (13), if $u \Rightarrow v$ is in $E_{C}$, then $\operatorname{Repr}(u) \in \operatorname{Repr}(v)$. Next we prove that if $\operatorname{Repr}(u) \in \operatorname{Repr}(v), u, v \in N_{C}$, then the edge $u \Rightarrow v$ is in $E_{C}$. If height $(u)=0$, then $\operatorname{Repr}(u)=I(u)$, and by (13) $|\operatorname{Repr}(u)| \leq 1$. On the other hand, as observed above, if height $(u) \neq 0$, then $|\operatorname{Repr}(u)| \neq 3$. In any case $|\operatorname{Repr}(u)| \neq 3$, for all $u \in N_{C}$. Thus $\operatorname{Repr}(u) \notin I(v)$ and by (14) there must exist $w$ such that $w \Rightarrow v$ is in $E_{C}$ and $\operatorname{Repr}(u)=\operatorname{Repr}(w)$. But by (a) above $u=w$, hence $u \Rightarrow v$ is in $E_{C}$, proving (b) and in turn completing the proof of the lemma.

Next we state some properties of the map Repr.

LEMMA $3.9 \quad$ (a) $\operatorname{Repr}\left(v^{\prime}\right)=\{\operatorname{Repr}(v)\}$, for all $v^{\prime} \in C l_{1}$;

(b) $\operatorname{Repr}\left(\operatorname{node}\left(q_{0}\right)\right)=\emptyset$

(c) $\operatorname{Repr}\left(v_{L}\right)=\{\emptyset,\{\operatorname{Repr}(v)\}\}$, for all $v_{L} \in \mathrm{Cl}_{2}$;

(d) $\operatorname{Repr}(\underline{2})=\{\emptyset,\{\emptyset\}\}=2$;

(e) $\operatorname{Repr}\left(v_{R}\right)=\{2,\{\operatorname{Repr}(v)\}\}$, for all $v_{R} \in \mathrm{Cl}_{3}$;

(f) $|\operatorname{Repr}(v)| \geq \omega$, for all $v \in C l_{4} \backslash\left\{\operatorname{node}\left(q_{0}\right)\right\}$; 
(g) $|\operatorname{Repr}(v)|=1$, for all $c \in C_{5}$;

(h) $\operatorname{Repr}\left(u_{v, w}\right)=[\operatorname{Repr}(v), \operatorname{Repr}(w)]$, for all $u_{v, w} \in \mathrm{Cl}_{6}$.

Proof. The lemma follows from Lemmas 3.5, 3.6, 3.8 and the definition (14) of Repr.

An immediate consequence of the preceding lemma is the following result.

COROLLARY 3.10 If $[s, t] \in \operatorname{Repr}(u)$, with $u \in N_{C}$, then there exist $u_{v, w} \in$ $\mathrm{Cl}_{6}$ such that the edge $u_{v, w} \Rightarrow u$ is in $E_{C}$ and $\operatorname{Repr}\left(u_{v, w}\right)=[s, t]$.

For each variable $x$ occurring in $P$, put

$$
M^{*} x=\operatorname{Repr}(\operatorname{node}(x)) \text {. }
$$

Also, for each $\pi \in \Pi$ we put

$$
\bar{\pi}=\operatorname{Repr}(\operatorname{node}(\pi)) \text {. }
$$

We will prove below that $M^{*}$ is an injective model of the conjunction $P$. We need the following lemma.

LEMMA 3.11 (a) The sets $\bar{\pi}$ are nonempty and pairwise disjoint.

(b) For each variable $x$ occurring in $P, M^{*} x=\bigcup_{\pi(x)=1} \bar{\pi}$.

(c) For every variable $x \in V \backslash\{U\}, M^{*} x \in \overline{\pi^{x}}$.

(d) For all variables $x, y \in V \backslash\{U\},\left[M^{*} x, M^{*} y\right] \in \overline{\pi^{x, y}}$.

(e) If $[s, t] \in \bar{\alpha}$, with $\alpha \in \Pi$, then for some $\beta \in \operatorname{dom}(\alpha), s \in \bar{\beta}$.

(f) If $s \in \bar{\beta}$, with $\beta \in \Pi$, then there is a $D$-pair $(\Gamma, \beta)$ such that for each $\gamma \in \Gamma, s \in \operatorname{Dom}(\bar{\gamma})$.

(g) If $[s, t] \in \bar{\alpha}$, with $\alpha \in \Pi$, then for some $\beta \in \operatorname{inv}(\alpha),[t, s] \in \bar{\beta}$.

(h) If $\left[s, t_{1}\right],\left[s, t_{2}\right] \in M^{*} f$, for some variable $f$ such that the conjunct SINGLEVALUED $(f)$ is in $P$, then $t_{1}=t_{2}$.

Proof. (a) By Lemma $3.9(\mathrm{f}), \bar{\pi}=\operatorname{Repr}(\operatorname{node}(\pi))$ is infinite. The pairwise disjointness of sets $\bar{\pi}, \pi \in \Pi$, follows from Lemma 3.2(a),(b), the quasiextensionality of $G_{C}$ (cf. Lemma 3.6) and Lemma 3.8.

(b) This is an immediate consequence of Lemma $3.7(f)$ and the definition of $\operatorname{Repr}$ (cf. (14)). 
(c) Lemma 3.7(a) yields that for each $x \in V \backslash\{U\}$ the edge node( $x) \Rightarrow$ $\operatorname{node}\left(\pi^{x}\right)$ is in $E_{C}$. Therefore

$$
M^{*} x=\operatorname{Repr}(\operatorname{node}(x)) \in \operatorname{Repr}\left(\operatorname{node}\left(\pi^{x}\right)\right)=\overline{\pi^{x}} .
$$

(d) Let $x, y \in V \backslash\{U\}$. Then by Lemma 3.7(b) and Lemma 3.9(h) we have

$$
\left[M^{*} x, M^{*} y\right]=[\operatorname{Repr}(\operatorname{node}(x)), \operatorname{Repr}(\operatorname{node}(y))] \in \overline{\pi^{x, y}} .
$$

(e) Assume that $[s, t] \in \bar{\alpha}$, for some $\alpha \in \Pi$. Then by Corollary 3.10 there exists $u_{v, w} \in C l_{6}$ such that $u_{v, w} \Rightarrow \operatorname{node}(\alpha)$ is in $E_{C}$, and $\operatorname{Repr}\left(u_{v, w}\right)=[s, t]$. Let $\beta$ be the place in $\Pi$ such that $v \Rightarrow \operatorname{node}(\beta)$ is in $E_{C}$. By Lemma 3.7(c) there exists a $D$-pair $(\Gamma, \beta)$ such that in particular $\alpha \in \Gamma$. Hence by the definition itself of a $D$-pair (cf. Definition 2.5(ii)), $\beta \in \operatorname{dom}(\alpha)$. In addition, since $v$ is connected to $\operatorname{node}(\beta)$ in $G_{C}$, it follows that $\operatorname{Repr}(v) \in \bar{\beta}$. But $[s, t]=\operatorname{Repr}\left(u_{v, w}\right)=$ $[\operatorname{Repr}(v), \operatorname{Repr}(w)](\mathrm{cf}$. Lemma 3.9(h)), hence $s \in \bar{\beta}$ with $\beta \in \operatorname{dom}(\alpha)$, proving (e).

(f) Next, suppose that $s \in \bar{\beta}$, for some place $\beta \in \Pi$. Hence $s=\operatorname{Repr}(v)$ with $v \Rightarrow \operatorname{node}(\beta)$ in $E_{C}$. Again by Lemma $3.7(\mathrm{c})$ there exists a $D$-pair $(\Gamma, \beta)$ such that for each $\gamma$ there is a node $w_{\gamma}$ for which $u_{v, w_{\gamma}} \Rightarrow \operatorname{node}(\gamma)$ is in $E_{C}$. Hence, by Lemma 3.9(h), $\left[s, \operatorname{Repr}\left(w_{\gamma}\right)\right] \in \bar{\gamma}$, which in turn implies $s \in \operatorname{Dom}(\bar{\gamma})$.

(g) Let $[s, t] \in \bar{\alpha}$, with $\alpha \in \Pi$. Following the proof of (e) above, we have that there is a node $u_{v, w}$ in $C l_{6}$ such that $u_{v, w} \Rightarrow \operatorname{node}(\alpha)$ is in $E_{C}$ and $\operatorname{Repr}\left(u_{v, w}\right)=$ $[s, t]$ (which by Lemma $3.9(\mathrm{~h})$ implies $s=\operatorname{Repr}(v)$ and $t=\operatorname{Repr}(w)$ ). From Lemma $3.7(\mathrm{e})$, it follows that $u_{w, v} \Rightarrow \operatorname{node}(\beta)$ is in $E_{C}$ for some $\beta \in \operatorname{inv}(\alpha)$. Hence $[t, s]=[\operatorname{Repr}(w), \operatorname{Repr}(v)] \in \bar{\beta}$, with $\beta \in \operatorname{inv}(\alpha)$.

(h) Finally, let $\left[s, t_{1}\right],\left[s, t_{2}\right] \in M^{*} f$, where the clause SINGLEVALUED $(f)$ is in $P$. Let $\left[s, t_{1}\right] \in \bar{\alpha}_{1}$ and $\left[s, t_{2}\right] \in \bar{\alpha}_{2}$, with $\alpha_{1}, \alpha_{2} \subseteq f$. Corollary 3.10 and Lemma 3.8(a) give that $\left[s, t_{1}\right]=\operatorname{Repr}\left(u_{v_{1} w_{2}}\right)$ and $\left[s, t_{2}\right]=\operatorname{Repr}\left(u_{v, w_{2}}\right)$, for some nodes $v, w_{1}, w_{2}$ such that $u_{v, w_{1}} \Rightarrow \operatorname{node}\left(\alpha_{1}\right)$ and $u_{v, w_{2}} \Rightarrow \operatorname{node}\left(\alpha_{2}\right)$ are in $E_{C}$. Hence by Lemma $3.7(\mathrm{c})$, there is a $D$-pair $\left(\Gamma,{ }^{v} \pi\right)$ such that $\alpha_{1}, \alpha_{2} \in \Gamma$ and $v \Rightarrow \operatorname{node}\left({ }^{v} \pi\right)$ is in $E_{C}$. But then the definition itself of a $D$-pair (cf. Definition 2.5(iii)) implies $\alpha_{1}=\alpha_{2}$, which by Lemma 3.7(d) in turn gives $v, w_{1}, w_{2} \in$ $\{\operatorname{node}(x): x \in V \backslash\{U\}\}$. Let $v=\operatorname{node}(x), w_{1}=\operatorname{node}(y)$, and $w_{2}=\operatorname{node}(z)$. Then, by Lemma 3.7(b), $\pi^{x, y}=\alpha_{1}=\alpha_{2}=\pi^{x, z}$, which, by condition C11, yields $y=z$. Hence $t_{1}=\operatorname{Repr}\left(w_{1}\right)=\operatorname{Repr}(\operatorname{node}(y))=\operatorname{Repr}\left(w_{2}\right)=t_{2}$, which concludes the proof of $(h)$ and in turn of the lemma.

Now we are ready to prove that the assignment $M^{*}$ is an injective model of all conjuncts in $P$. 
As concerns the injectivity, notice that by condition C1 the map $x \mapsto\{\pi \in$ II : $\pi \subseteq x\}$ is one-one. Thus Lemma 3.11(a),(b) yields that $M^{*} x \neq M^{*} y$ for any two distinct variables $x$ and $y$ in $P$, i.e. $M^{*}$ is injective.

Next we prove that all conjuncts of $P$ are satisfied by the assignment $M^{*}$.

Let $x=y \cup z$ be in $P$. Then in view of Lemma 3.11(a),(b) and the properties of places (cf. Definition 2.2), we have

$$
M^{*} x=\bigcup_{\pi(x)=1} \bar{\pi}=\bigcup_{\substack{\pi(y)=1 \\ \gamma \pi(z)=1}} \bar{x}=\bigcup_{\pi(y)=1} \bar{\pi} \cup \bigcup_{\substack{\pi(z)=1\\}} \bar{\pi}=M^{*} y \cup M^{*} z,
$$

i.e. $x=y \cup z$ is modeled correctly by $M^{*}$.

Next, let $x=y \backslash z$ be in $P$. Then we have

$$
M^{*} x=\bigcup_{\pi(x)=1} \bar{\pi}=\bigcup_{\substack{\pi(y)=1 \\ \lambda \pi(z)=0}} \bar{\pi}=\bigcup_{\pi(y)=1} \bar{\pi} \backslash\left(\bigcup_{\pi(z)=1} \bar{\pi}\right)=M^{*} y \backslash M^{*} z
$$

which shows that the conjunct $x=y \backslash z$ is also satisfied by $M^{*}$.

If $x \in y$ (resp. PAIR_IN $(x, y, f)$ ) occurs in $P$, then since $\pi^{x}$ (resp. $\pi^{x, y}$ ) is a place at the variable $x$ (resp. at the pair $[x, y]$ ), then (cf. Definition 2.3 (resp. Definition 2.4)) $\pi^{x}(y)=1\left(\right.$ resp. $\left.\pi^{x, y}(f)=1\right)$. Therefore Lemma 3.11(c) (resp. Lemma $3.11(\mathrm{~d})$ ) yields $M^{*} x \in \bar{\pi}^{*} \subseteq M^{*} y$, i.e. $M^{*} x \in M^{*} y$ (resp. $\left.\left[M^{*} x, M^{*} y\right] \in M^{*} f\right)$. Ilence literals of type $x \in y($ resp. PAIR $I N(x, y, f))$ are correctly modeled by $M^{*}$.

Next, let $d=D f$ be a clause in $P$. In this case we need to verify that $M^{*} d=\operatorname{Dom}\left(M^{*} f\right)$, which we do as follows. Let $s \in M^{*} d$, and in particular let $\beta \subseteq d$ be such that $s \in \bar{\beta}$. From Lemma 3.11(f) it follows that there is a $D$-pair $(\Gamma, \beta)$ such that for each $\gamma \in \Gamma, s \in \operatorname{Dom}(\bar{\gamma})$. Also, from the definition itself of a $D$-pair (cf. Definition 2.5(iv)), since $\beta(d)=1$, there is a $\gamma_{0} \in \Gamma$ such that $\gamma_{0}(f)=1$, where the clause $d=D f$ is in $P$. Hence $s \in \operatorname{Dom}\left(\bar{\gamma}_{0}\right) \subseteq \operatorname{Dom}\left(M^{*} f\right)$, which in turn implies $M^{*} d \subseteq \operatorname{Dom}\left(M^{*} f\right)$. To prove the converse inclusion, let $s \in \operatorname{Dom}\left(M^{*} f\right)$. Thus for some set $t,[s, t] \in M^{*} f$. Let $\alpha \subseteq f$ such that $[s, t] \in \bar{\alpha}$. Lemma 3.11(e) yields the existence of a place $\beta \in \operatorname{dom}(\alpha)$ such that $s \in \bar{\beta}$. But then from condition C3(ii), we have $\beta(d)=1$, which implies $s \in M^{*} d$. Thus $\operatorname{Dom}\left(M^{*} f\right) \subseteq M^{*} d$, and in conclusion $M^{*} d=\operatorname{Dom}\left(M^{*} f\right)$, proving that cluases $d=D f$ are correctly modeled too.

As regards clauses of type $\operatorname{INV}$, let $\operatorname{INV}(f, g)$ be one such. Assume that $[s, t] \in M^{*} f$ and let $\alpha \subseteq f$ be such that $[s, t] \in \bar{\alpha}$. Then, from Lemma 3.11(g), there exists a place $\beta \in \operatorname{inv}(\alpha)$ such that $[s, t] \in \bar{\beta}$. To prove that $[t, s] \in M^{*} g$, it only remains to show that $\beta(g)=1$. But this follows immediately from 
condition $\mathrm{C} 4(\mathrm{iii})$. Symmetrically one can prove that if $[s, t] \in M^{*} g$, then $[t, s] \in$ $M^{*} f$. Hence $M^{*} f$ is an inverse of $M^{*} g$, in the sense specified at the beginning of Section 1.

Finally, from Lemma $3.11(\mathrm{~h})$ it follows that $M^{*}$ satisfies all conjuncts in $P$ of the form SINGLEVALUED $(f)$.

This concludes the proof that $M^{*}$ is an injective model of $P$. Thus we have

LEMMA 3.12 The conditions stated in Lemma 2.7, necessary for the injective satisfiability of a conjunction $P$ of simple positive clauses of type $x=y \cup z$, $x=y \backslash z, x \in y, \operatorname{PAIR} I N(x, y, f), d=D f, \operatorname{INV}(f, g), \operatorname{SINGLEVAlUED}(f)$, containing clauses (3), are also sufficient.

In view of Lemma 1.1 and by observing that the conditions stated in Lemma 2.7 are effectively verifiable we have

THEOREM 3.13 The class $\mathcal{L}$ of propositional combinations of set-theoretic clauses of the form (1) has a solvable satisfibility problem, and Lemma 3.12 suggests a specific decision procedure for $\mathcal{L}$.

\section{Acknowledgements}

The authors would like to thank E. Omodeo and A. Ferro for helpful discussions and comments.

This work was partially supported by ENI and ENIDATA within the AXL project and by the U.S. NSF grant \# DCR-84-01633.

\section{References}

[Can] Cantone,D., Decision procedures for elementary sublanguages of set theory.X.Multilevel syllogistic extended by the powerset and singleton operators, this volume.

[CFS] Cantone,D., Ferro,A., and Schwartz,J.T., Decision procedures for elementary sublanguages of set theory. $V$. Multilevel syllogistic extended by the general union operator. Jour. Comp. Syst. Sci., 34,1 (1987).

[CGO] Cantone,D., Ghelfo,S., and Omodeo,E.G., The automation of syllogistic. I. Syllogistic normal forms, Journ. Symb., Comp., to appear (1987). 
[FOS] Ferro,A., Omodeo,E., and Schwartz,J.T., Decision procedures for elementary fragments of set theory, Fifth Conf. on Automated Deduction, Les Arcs, France, Lect. Notes in Comp. Sci. 87, Springer-Verlag.

[Jec] Jech, T., Set Theory, Academic Press, New York, 1978.

[PaP] Parlamento, F., and Policriti, A., On the representability of well founded graphs and the axion of choice, to appear. 
NYU COMPSCI TR-374

C. 2

Cantone, D

Decision procedures for

elementary sublanguages of set theory. XI.

7 NYU COMPSCI TR-374

Cantone, D

Decision procedures for

elementary sublanguages of

$\Rightarrow$ set theory. XI.

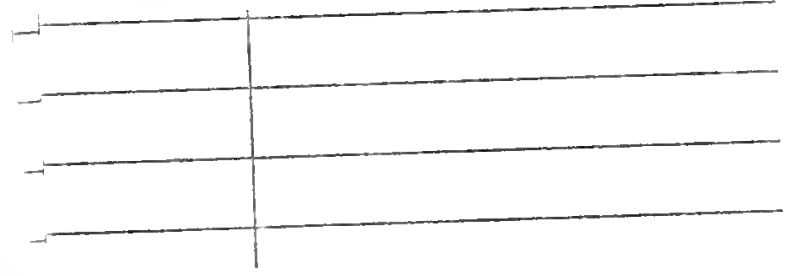

This book may be kert
EOURTEEN DA

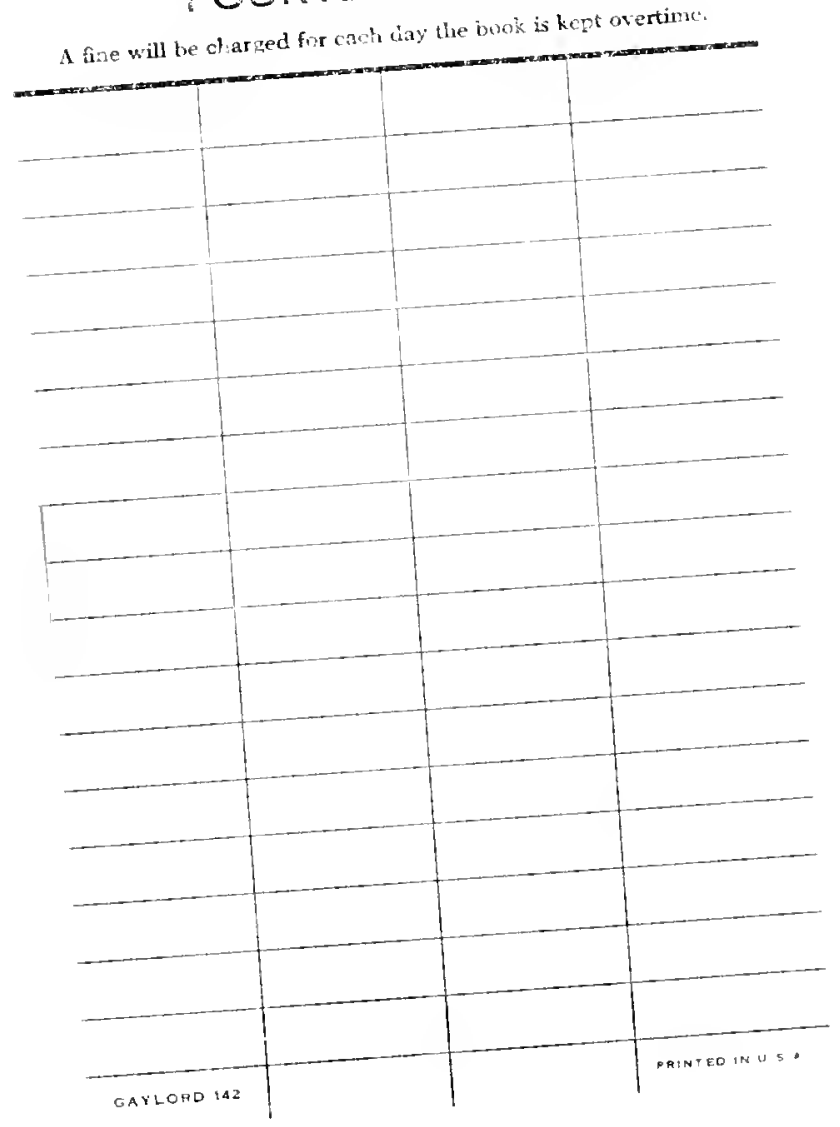


